\title{
Microturbellarians (Platyhelminthes and Acoelomorpha) in Brazil: invisible organisms?
}

\author{
J. A. L. Braccini ${ }^{a, b}$, S. V. Amaral ${ }^{a, b}$ and A. M. Leal-Zanchet ${ }^{a, b *}$ \\ anstituto de Pesquisas de Planárias, Universidade do Vale do Rio dos Sinos - UNISINOS, \\ Avenida Unisinos, 950, CEP 93022-000, São Leopoldo, RS, Brazil \\ 'Programa de Pós-graduação em Biologia, Universidade do Vale do Rio dos Sinos - UNISINOS, \\ Avenida Unisinos, 950, CEP 93022-000, São Leopoldo, RS, Brazil \\ *e-mail: zanchet@unisinos.br
}

Received: October 10, 2014 - Accepted: March 4, 2015 - Distributed: May 31, 2016

(With 1 figure)

\begin{abstract}
Microturbellarians typically belong to the benthos and may occur in a wide variety of environments. They are abundant in freshwater and marine ecosystems and may occur in moist terrestrial habitats. However, turbellarians are seldom taken into account in studies of biodiversity. Most studies on Brazilian microturbellarians had taxonomical purposes and were done in the years 1940-1950. Thus, information on their occurrence and ecological aspects are dispersed throughout several papers. We intend here to summarize the biogeographical distribution and ecological aspects of microturbellarians recorded for Brazil, indicating the main gaps in their knowledge and possible actions to enhance studies on this group. There are 239 species of microturbellarians registered for Brazil, with records distributed in 12 states. However, just three states located in southern Brazil have records of $94 \%$ of microturbellarian species. Thus, knowledge on the systematics and geographical distribution of Brazilian microturbellarians clearly reflect the scientific activity over many years or decades in two states of southeastern and southern Brazil. Considering the scant information on this group in Brazil, which is also the situation of the Neotropical microturbellarians in general, some actions should be proposed. First, it would be necessary to sample in the diverse biomes, as well as in the various river and sea basins, based on standardized sampling protocols. Second, it would be necessary to encourage diverse research groups to include microturbellarians and/or turbellarians in general into biodiversity inventories and studies on community structure of invertebrates. Third, it is necessary to increase the number of research groups on microturbellarians, in order to augment the studies on their morphology, systematics, and ecology. Considering their abundance, species richness and ecological importance in aquatic environments, despite some peculiarities regarding their sampling, sorting and identification procedures, the challenge to study microturbellarians and enhance knowledge about them in Brazilian ecosystems should be faced.
\end{abstract}

Keywords: Platyhelminthes, species diversity, Neotropical region, systematics, ecology.

\section{Microturbelários (Platyhelminthes e Acoelomorpha) no Brasil: organismos invisíveis?}

\section{Resumo}

Microturbelários são tipicamente bentônicos e podem ocorrer em uma ampla variedade de ambientes. São abundantes em ecossistemas marinhos e de água doce, podendo ocorrer em ambientes terrestres úmidos. Entretanto, turbelários raramente são considerados em estudos de diversidade. A maioria dos estudos sobre microturbelários brasileiros tiveram propósito taxonômico e foram realizados nos anos 1940-1950. Assim, informações sobre ocorrência e aspectos ecológicos estão dispersos em diversos artigos. O objetivo deste trabalho é sumarizar a distribuição biogeográfica e aspectos ecológicos dos microturbelários registrados para o Brasil, indicando as principais lacunas do conhecimento e possíveis ações para ampliar estudos sobre esse grupo. Há 239 espécies de microturbelários registradas no Brasil, com registros distribuídos em 12 estados. No entanto, $94 \%$ das espécies de microturbelários foram registradas em apenas três estados localizados no sul e sudeste do Brasil. Assim, o conhecimento sobre a sistemática e distribuição geográfica dos microturbelários claramente reflete as atividades científicas realizadas por muitos anos ou mesmo décadas em dois estados do sudeste e sul do Brasil. Considerando as escassas informações existentes sobre esse grupo no Brasil, assim como a situação dos microturbelários neotropicais em geral, algumas ações devem ser propostas. Primeiramente, é necessário realizar amostragens em diversos biomas, assim como nas várias bacias e regiões hidrográficas marinhas, baseadas em protocolos de amostragem padronizados. Em segundo lugar, faz-se necessário incentivar diversos grupos de pesquisa a incluir 
microturbelários e/ou turbelários em geral em inventários da biodiversidade e estudos de estrutura de comunidades de invertebrados. Em terceiro lugar, é necessário ampliar o número de grupos de pesquisa em microturbelários, para aumentar os estudos sobre sua morfologia, sistemática e ecologia. Considerando sua abundância, riqueza de espécies e importância ecológica em ambientes aquáticos, apesar de suas peculiaridades de amostragem, triagem e identificação, o desafio de estudar e ampliar o conhecimento sobre microturbelários em ecossistemas brasileiros deve ser enfrentado.

Palavras-chave: Platyhelminthes, diversidade de espécies, região Neotropical, sistemática, ecologia.

\section{Introduction}

Turbellarians are acoelomate, soft-bodied worms that have a sac-like gut and typically ciliated epidermal cells. Most turbellarians are hermaphrodites with cross fertilization following copulation, showing a complex reproductive system (Cannon, 1986; Rieger et al., 1991). Traditionally, flatworms used to belong to the phylum Platyhelminthes, which can be subdivided into three clades, viz. Acoelomorpha, Catenulida e Rhabditophora (Rieger et al., 1991), since Rhabditophora also includes the parasitic forms. The Acoelomorpha, however, has been removed from the Platyhelminthes into its own phylum (Baguñà and Riutort, 2004; Tyler et al., 2014; Littlewood, 2006; Larsson and Jondelius, 2008; Boll et al., 2013). The term turbellarians continues to be used and will be applied here without taxonomic connotation.

There are about 6,500 species of turbellarians worldwide (Schockaert et al., 2008). They are distributed into the following taxa: Acoela, Nemertodermatida, Catenulida, Macrostomida, Polycladida, Prolecithophora, Lecithoepitheliata, Revertospermata, Proseriata, Tricladida, Dalytyphloplanida and Kalyptorhynchia (Schockaert et al., 2008; Van Steenkiste et al., 2013). The later two constitute the rhabdocoels. Turbellarians can be divided into two major groups, with no taxonomical association: the macroturbellarians or large worms, which include triclads and polyclads, and the microturbellarians including the other turbellarian groups, the members of which are smaller.

Turbellarians may occur in a wide variety of environments. They are abundant in freshwater and marine ecosystems and may occur in moist terrestrial habitats. They are, however, mainly marine animals, with only $1 / 5$ of the known species occurring in freshwater (Hyman, 1951; Schockaert et al., 2008). Turbellarians typically belong to the benthos. Triclads and polyclads occur generally on hard bottom, under stones, or associated with macrophytes, algae or gravel (Hyman, 1951; Du-Bois Reymond Marcus and Marcus, 1968). Microturbellarians usually occur on sandy or muddy bottom. Most marine turbellarians are limited to the littoral zone of the ocean (Hyman, 1951). Freshwater forms may occur in temporary or permanent wetlands, such as lakes, natural or artificial ponds, pools, ditches, streams and rivers (Kolasa, 1991; Noreña-Janssen, 1995; Young, 2001), as well as in agroecosystems (Bambaradeniya et al., 2004; Vara and Leal-Zanchet, 2013). Some marine flatworms, such as acoels and polyclads, and freshwater rhabdocoels are planctonic (Hyman, 1951; Rocha et al., 1990; Dumont et al., 2014). Some microturbellarians may occur in humid terrestrial environments (Hyman, 1951; Tessens et al., 2014); land triclads may be a species-rich group in tropical and subtropical ecosystems (Carbayo et al., 2009). The temnocephalids, a group of the taxon Dalytyphloplanida, and some members of other taxonomic groups of microturbellarians live in association with other invertebrates or with turtles, usually as ectosymbionts (Hyman, 1951; Jennings, 1997). In addition, some acoels and rhabdocoels are parasites of molluscs, echinoderms and other invertebrates (Hyman, 1951; Bush, 1981).

Microturbellarians in general were studied mainly in Brazil in the years 1940-1950 (Marcus, 1943, 1944, 1945a, b, 1946, 1948, 1949, 1950, 1951, 1952, 1954; Du-Bois Reymond Marcus, 1951; Marcus and Macnae, 1954), based on samplings in the northern littoral of the state of São Paulo and lentic environments of São Paulo city and its neighbourhood. Later, Rocha et al. (1990) and Hooge and Rocha (2006) studied, respectively, freshwater rhabdocoels and acoels from the state of São Paulo. In addition, a new species of marine rhabocoel was recently described (Reygel et al., 2014). Freshwater turbellarians from southern Brazil were investigated by Gamo and Leal-Zanchet (2004), Vara and Leal-Zanchet (2013) and Braccini and Leal-Zanchet (2013) in natural wetlands and agroecosystems. Temnocephalids from various Brazilian regions were studied by Monticelli (1899), Pereira and Cuocolo (1940, 1941), Amato et al. (2003, 2005, 2006, 2007, 2010, 2011), Amato and Amato (2005) and Seixas et al. (2010a, b, c, 2011, 2014).

Freshwater and land triclads were studied mainly in the Brazilian states of São Paulo and Rio Grande do Sul (Carbayo and Froehlich, 2008; Baptista et al. 2010; Leal-Zanchet et al., 2011; Amaral et al., 2014). Polyclads were mainly known from the coast of the states of São Paulo and Rio de Janeiro, as well as the northeastern coast of Brazil (Carbayo and Froehlich, 2008; Bahia and Padula, 2009; Bahia et al., 2012; Bulnes and Torres, 2014).

In contrast to triclads and polyclads, which are collected directly, microturbellarians should be sampled with some substratum using a fine-meshed sweep-net (Young, 2001). Material collected by the net should be transferred to plastic vials containing water from the sampling site. The vials should be transported to the laboratory and carefully examined under a stereomicroscope (Braccini and Leal-Zanchet, 2013; Vara and Leal-Zanchet, 2013). For sorting, samples may be treated by the method of reduction of oxygen (Schockaert, 1996) or by examining all sampling water under the stereomicroscope (Brusa et al., 2003). Live turbellarians should be transferred by a pipette to a petri dish and be examined alive for observation of general shape, size and colour pattern. After that, the 
internal morphology should be examined using a "squeeze preparation" technique (Gamo, 1987; Young, 2001).

Considering that the macroturbellarians are the best known group among turbellarians and that their study in Brazil was summarized by Carbayo and Froehlich (2008), we will focus on Brazilian microturbellarians herein. We intend to summarize the biogeographical distribution and ecological aspects of microturbellarians recorded for Brazil, by pointing out main gaps in the knowledge about them and possible actions to enhance studies on this group.

\section{Methods}

Original research articles were searched in the databases Thomson Reuters (ISI) and Scielo, between August 2014 and February 2015, and in the bibliographical assets of our laboratory in the Universidade do Vale do Rio dos Sinos (UNISINOS). The following key-words were used: Platyhelminthes, Acoela, Nemertodermatida, Catenulida, Prolecithophora, Rhabdocoela, Temnocephalida, Macrostomida, Lecithoepitheliata or Revertospermata and Brazil.

We organized the data thus obtained in order to summarize the following information: species, order or other representative taxonomic group, sampling place (city and state), and type of environment where the occurrence was recorded. Regarding the environment, the following data were considered: type of substratum (sand, mud, algae etc.), type of ecosystem (marine, freshwater or brackish water) and hydric regime (lentic or lotic). In the case of temnocephalids, the associated organism was informed. The classification of marine ecoregions follows Spalding et al. (2007).

\section{Geographical Distribution and Ecological Aspects}

A total of 44 articles with records of microturbellarians in Brazil was found, 26 of which were available on the online databases Thomson Reuters (ISI) and Scielo. Eighteen other articles were found in the bibliographical assets of our laboratory (UNISINOS).

There are 239 species of microturbellarians registered for Brazil, with records distributed in 12 states. The highest number of species was recorded for the state of São Paulo, followed by Rio Grande do Sul and Paraná (Table 1). The central, northern and northeastern regions of Brazil have no or just a few records (Figure 1).

Brazilian microturbellarians are distributed among 10 taxonomical groups. Dalytyphloplanida and Catenulida are the most species-rich, with about $28 \%$ and $18 \%$ of the recorded species, respectively, followed by Acoela (15\%), Prolecithophora (11\%), Proseriata (10\%), Kalyptorhynchia (9\%), and Macrostomida (7\%). Lecithoepitheliata and Revertospermata are taxons with only few known species (Tables 1 and 2). The taxon Nemertodermatida has no records in Brazil.

Acoels, a group of marine flatworms, are represented by specimens sampled only in the state of São Paulo, mainly in sandy beaches, on algae or rock coasts (Tables 1 and 2). A few specimens were found on beach ponds.

Catenulids, which are mainly freshwater inhabitants, were registered in various continental environments, mainly in southeastern and southern Brazil (Table 1). They were frequently found in lentic environments, including rice fields and their canals, or in humid terrestrial environments. Six species were found in lotic environments and two species in water accumulated among leaves of Bromeliaceae (Table 3).

Table 1. Brazilian states with records of microturbellarians and number of species recorded per taxonomical group and state.

\begin{tabular}{|c|c|c|c|c|c|c|c|c|c|c|c|c|c|}
\hline \multirow[b]{2}{*}{ Taxon } & \multicolumn{3}{|c|}{ Northern region } & \multicolumn{2}{|c|}{$\begin{array}{c}\text { Central } \\
\text { region }\end{array}$} & \multicolumn{4}{|c|}{ Southeastern region } & \multicolumn{4}{|c|}{ Southern region } \\
\hline & 旁 & 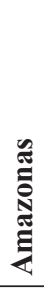 & อี & 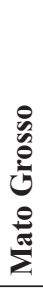 & 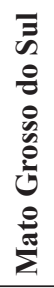 & 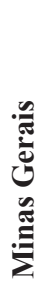 & 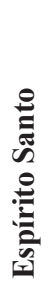 & 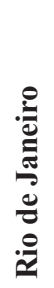 & 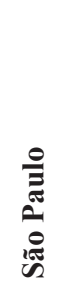 & صَّ & 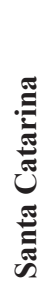 & 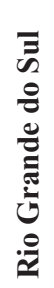 & 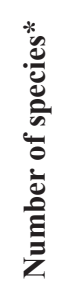 \\
\hline Acoela & - & - & - & - & - & - & - & - & 36 & - & - & - & 36 \\
\hline Catenulida & - & - & 1 & - & - & - & - & - & 41 & 13 & - & 21 & 42 \\
\hline Dalytyphloplanida & 2 & 1 & 1 & 1 & 1 & 7 & 1 & 1 & 45 & 3 & 1 & 24 & 68 \\
\hline Kalyptorhynchia & - & - & - & - & - & - & - & - & 20 & - & - & 1 & 21 \\
\hline Lecithoepitheliata & - & - & - & - & - & - & - & - & 2 & 2 & - & 1 & 2 \\
\hline Macrostomida & - & - & - & - & - & - & - & - & 15 & - & - & 4 & 17 \\
\hline Prolecithophora & - & - & - & - & - & - & - & - & 27 & - & - & - & 27 \\
\hline Proseriata & - & - & - & - & - & - & - & 2 & 24 & - & - & 1 & 25 \\
\hline Revertospermata & - & - & - & - & - & - & - & - & 1 & - & - & - & 1 \\
\hline Number of species & 2 & 1 & 2 & 1 & 1 & 7 & 1 & 3 & 211 & 18 & 1 & 52 & 239 \\
\hline
\end{tabular}

- No data. *Species may occur in more than one locality. 


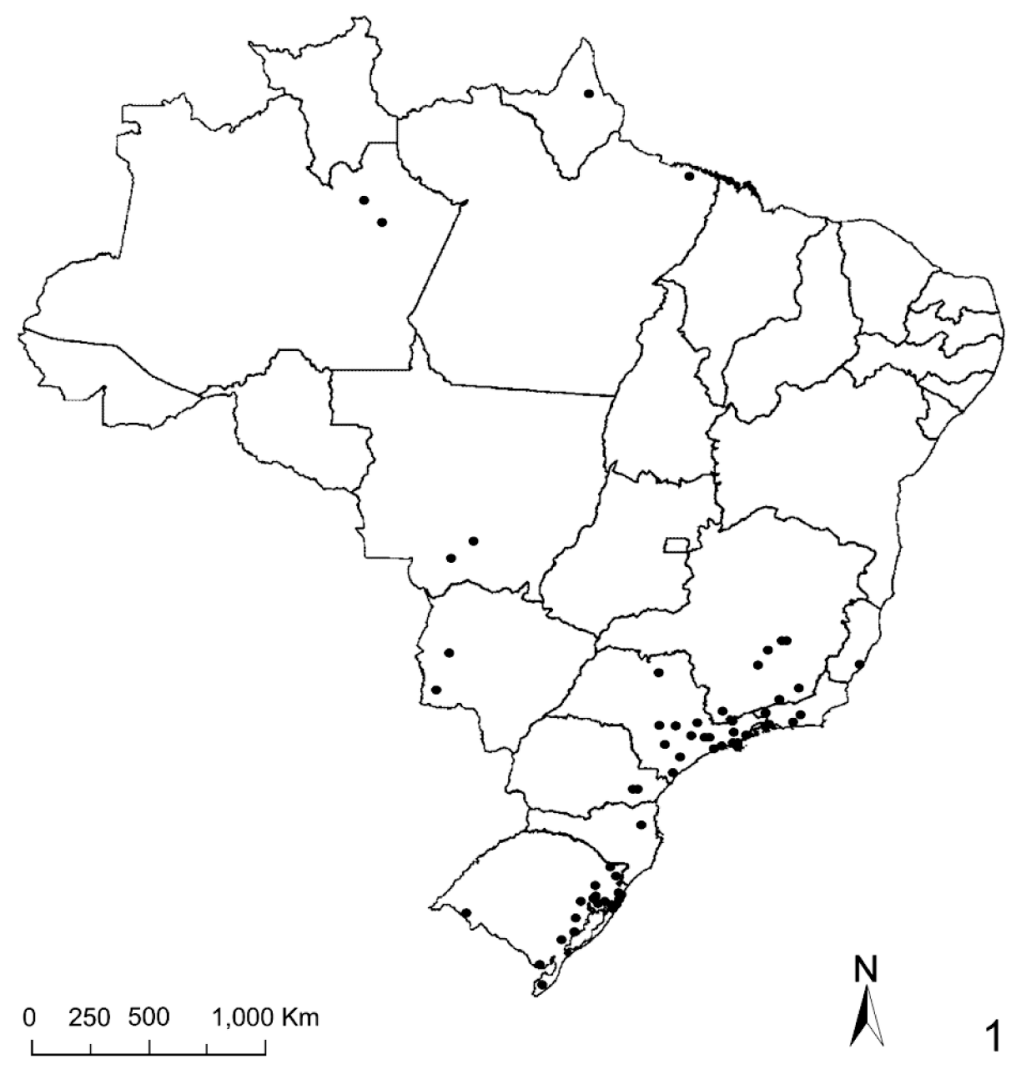

Figure 1. Records of microturbellarians in Brazil. Dots represent localities where microturbellarians were sampled.

Macrostomids inhabit marine and freshwater environments. They occur associated to the bottom or to the vegetation. In Brazil, 11 species were recorded in marine environments of the north coast of the state of São Paulo. Six other species were sampled in lentic continental ecosystems or in brackish water in various regions (Tables 1 and 4).

Rhabdocoels (Dalytyphloplanida and Kalyptorhynchia) inhabit mainly marine and freshwater environments, but some species are terrestrial. In Brazil, marine species were recorded in the north coast of the state of São Paulo, on algae, sand or mud. Freshwater species were found in lentic ecosystems, including rice fields and their canals, or lotic environments of southeastern and/or southern Brazil. Temnocephalids occur only in freshwater environments, with most species recorded in Brazil. They were found in the north, center, southeast and south of Brazil, as ectosymbiotes in turtles, decapods, insects and molluscs (Tables 1 and 5).

Lecithopitheliates may occur in freshwater, terrestrial and marine environments, but they are represented in Brazil by only two freshwater species, Prorhynchus stagnalis Schultze, 1851 and Geocentrophora applanata (Kennel, 1888). Prorhynchus stagnalis, considered worldwide distributed, was recorded in various localities in southeastern and southern Brazil, whereas G. applanata was sampled in two localities of central and southeastern Brazil (Tables 1 and 6).

Prolecithophorans inhabit freshwater and marine environments, but they have no records in Brazilian freshwater ecosystems (Vara and Leal-Zanchet, 2013; Braccini and Leal-Zanchet, 2013). The members of this taxon were sampled in marine environments on coastal areas of the state of São Paulo (Table 1). They were found on algae (Padina and Ulva) or on sandy and rocky coasts (Tables 1 and 6).

Revertospermats are exclusively marine flatworms. They are represented in Brazil by a single species, Urastoma cyprinae (Graff, 1882), found on the coast of the state of São Paulo (Tables 1 and 6). It is a parasite which inhabits the gills of bivalves.

Proseriates may occur in marine and freshwater environments, but almost all Brazilian species were sampled mainly in the north coast of the state of São Paulo (Table 1). They were found mainly in small ponds with high organic matter content. Only Bothrioplana semperi Braun, 1881 was sampled in freshwater environments of southeastern and southern Brazil (Table 7). 
Table 2. Species of Acoela recorded for Brazil, with localities of occurrence and type of environment and/or substratum where the microturbellarians were found.

\begin{tabular}{|c|c|c|c|}
\hline Species & Locality & Environment & Substratum \\
\hline $\begin{array}{l}\text { Amphiscolops evelinae Marcus, } \\
1947\end{array}$ & Santos/SP; Ubatuba/SP & Marine & Among algae \\
\hline $\begin{array}{l}\text { Archaphanostoma marcusi Hooge } \\
\text { and Rocha, } 2006\end{array}$ & Ilhabela/SP & Marine & Sand \\
\hline $\begin{array}{l}\text { Avagina marci Dörjes e Karling, } \\
1975\end{array}$ & São Sebastião/SP; Ilhabela/SP & Marine & Sand \\
\hline Childia etium (Marcus, 1954) & São Sebastião/SP & Marine & Coarse sand \\
\hline $\begin{array}{l}\text { Childia groenlandica (Levinsen, } \\
\text { 1879) }\end{array}$ & Bertioga/SP & Mangrove & Mud \\
\hline Childia westbladi (Marcus, 1950) & Santos/SP; São Sebastião/SP & Marine & Among algae \\
\hline Convoluta hensen Bohmig, 1895 & Cananéia/SP; São Sebastião/SP & Marine & $*$ \\
\hline $\begin{array}{l}\text { Deuterogonaria thauma (Marcus, } \\
\text { 1952) }\end{array}$ & Santos/SP; São Sebastião/SP & & Among algae \\
\hline $\begin{array}{l}\text { Eumecynostomum evelinae } \\
\text { (Marcus, 1948) }\end{array}$ & $\begin{array}{c}\text { Guarujá/SP; Santos/SP; São } \\
\text { Sebastião/SP }\end{array}$ & Marine & $\begin{array}{l}\text { Among Ulva and } \\
\text { Enteromorpha/ mud }\end{array}$ \\
\hline Faerlea antora Marcus, 1952 & São Sebastião/SP & Marine & Mud \\
\hline $\begin{array}{l}\text { Haplocelis dichona (Marcus, } \\
\text { 1954) }\end{array}$ & São Sebastião/SP & Marine & Sand \\
\hline $\begin{array}{l}\text { Haplogonaria pellita (Marcus, } \\
\text { 1951) }\end{array}$ & São Sebastião/SP & Marine & Fine sand \\
\hline $\begin{array}{l}\text { Haplogonaria sophiae Hooge and } \\
\text { Rocha, } 2006\end{array}$ & São Sebastião/SP & Marine & Muddy sand \\
\hline $\begin{array}{l}\text { Haploposthia microphoca Marcus, } \\
1950\end{array}$ & São Sebastião/SP & Marine & Among algae \\
\hline $\begin{array}{l}\text { Heterochaerus carvalhoi (Marcus, } \\
\text { 1952) }\end{array}$ & Santos/SP & Brackish water & Muddy sand \\
\hline $\begin{array}{l}\text { Heterochaerus sargassi (Hyman, } \\
\text { 1939) }\end{array}$ & São Sebastião/SP & Marine & $\begin{array}{c}\text { Among algae Sargassum } \\
\text { sp./ on rock coasts }\end{array}$ \\
\hline Isodiametra divae (Marcus, 1950) & São Sebastião/SP; Ilhabela/SP & Marine & Muddy sand/coarse sand \\
\hline $\begin{array}{l}\text { Isodiametra westbladi (Marcus, } \\
\text { 1949) }\end{array}$ & $\begin{array}{c}\text { Santos/SP; São Sebastião/SP; } \\
\text { Ubatuba/SP; Caraguatatuba/SP; } \\
\text { Ilhabela/SP }\end{array}$ & Marine & Sand \\
\hline $\begin{array}{l}\text { Isodiametra vexillaria (Marcus, } \\
\text { 1948) }\end{array}$ & $\begin{array}{l}\text { Santos/SP; São Sebastião/SP; } \\
\text { Ilha das Palmas/SP }\end{array}$ & Marine & Sand \\
\hline Kuma albiventer (Marcus, 1954) & São Sebastião/SP & Marine & Muddy sand \\
\hline $\begin{array}{l}\text { Kuma asilhas Hooge and Rocha, } \\
2006\end{array}$ & São Sebastião/SP & Marine & Sand \\
\hline Kuma belca Marcus, 1952 & Ubatuba/SP & Marine & Sand \\
\hline Kuma brevicauda Marcus, 1950 & Cananéia/SP & Marine & Muddy sand \\
\hline $\begin{array}{l}\text { Mecynostomum tenuissimum } \\
\text { (Westblad, 1946) }\end{array}$ & São Sebastião/SP & Marine & Mud \\
\hline Nadina evelinae (Marcus, 1952) & São Sebastião/SP & Marine & Among algae \\
\hline $\begin{array}{l}\text { Otocelis erinae Hooge and Rocha, } \\
2006\end{array}$ & São Sebastião/SP; Ilhabela/SP & Marine & Fine sand \\
\hline $\begin{array}{l}\text { Paraproporus xanthus Marcus, } \\
1950\end{array}$ & São Sebastião/SP & Marine & Mud \\
\hline $\begin{array}{l}\text { Paratomella rubra } \text { Rieger and Ott, } \\
1971\end{array}$ & São Sebastião/SP & Marine & Fine sand \\
\hline
\end{tabular}

*Planctonic organism. 
Table 2. Continued...

\begin{tabular}{|c|c|c|c|}
\hline Species & Locality & Environment & Substratum \\
\hline $\begin{array}{l}\text { Philactinoposthia coneyi Hooge } \\
\text { and Rocha, } 2006\end{array}$ & Ilhabela/SP & Marine & Sand \\
\hline $\begin{array}{l}\text { Philactinoposthia stylifera } \\
\text { (Westblad, 1946) }\end{array}$ & São Sebastião/SP & Marine & Sand \\
\hline $\begin{array}{l}\text { Philocelis robrochai Hooge and } \\
\text { Rocha, } 2006\end{array}$ & Ilhabela/SP & Marine & Sand \\
\hline $\begin{array}{l}\text { Pseudactinoposthia daena } \\
\text { (Marcus, 1954) }\end{array}$ & Itanhaém/SP & Mangrove & Mud \\
\hline $\begin{array}{l}\text { Pseudanaperus tinctus (Marcus, } \\
\text { 1952) }\end{array}$ & São Sebastião/SP & Marine & Among algae \\
\hline $\begin{array}{l}\text { Pseudaphanostoma divae Marcus, } \\
1952\end{array}$ & Ubatuba/SP; São Sebastião/SP & Marine & Sand \\
\hline $\begin{array}{l}\text { Pseudaphanostoma herringi } \\
\text { Hooge and Rocha, } 2006\end{array}$ & Ilhabela/SP & Marine & Sand \\
\hline $\begin{array}{l}\text { Pseudokuma orphinum (Marcus, } \\
\text { 1950) }\end{array}$ & São Sebastião/SP & Marine & $\begin{array}{c}\text { Among Sargassum } \\
\text { stenophyllum on rock } \\
\text { coasts }\end{array}$ \\
\hline
\end{tabular}

*Planctonic organism.

Table 3. Species of Catenulida recorded for Brazil, with localities of occurrence and type of environment and/or substratum where the microturbellarians were found.

\begin{tabular}{|c|c|c|c|}
\hline Species & Locality & Environment & Substratum \\
\hline $\begin{array}{l}\text { Catenula alitha Marcus, } \\
1945\end{array}$ & São Paulo/SP; Paraná/PR & Freshwater (lentic) & - \\
\hline $\begin{array}{l}\text { Catenula evelinae (Marcus, } \\
\text { 1945) }\end{array}$ & São Paulo/SP & Freshwater (lentic) & - \\
\hline $\begin{array}{l}\text { Catenula lemnae Duges, } \\
1832\end{array}$ & $\begin{array}{c}\text { São Paulo/SP; Paraná/PR; São } \\
\text { Leopoldo/RS; Santo Antônio da } \\
\text { Patrulha/RS; Terra de Areia/RS; } \\
\text { Osório/RS; Capivari do Sul/RS; } \\
\text { Balneário Pinhal/RS; Tramandaí/RS; } \\
\text { Cachoeirinha/RS; Santo Antônio da } \\
\text { Patrulha/RS; Camaquã/RS }\end{array}$ & Freshwater (lentic) & $\begin{array}{l}\text { Sand and } \mathrm{mud} / \mathrm{on} \\
\text { macrophyte roots }\end{array}$ \\
\hline $\begin{array}{l}\text { Catenula leuca Marcus, } \\
1945\end{array}$ & São Paulo/SP & Freshwater (lentic) & - \\
\hline $\begin{array}{l}\text { Catenula macrura Marcus, } \\
1945\end{array}$ & São Paulo/SP & Freshwater (lentic) & - \\
\hline $\begin{array}{l}\text { Catenula sawayai (Marcus, } \\
\text { 1945) }\end{array}$ & São Paulo/SP; Paraná/PR & $\begin{array}{l}\text { Freshwater (lentic } \\
\text { and lotic) }\end{array}$ & - \\
\hline $\begin{array}{l}\text { Catenula turgida } \\
\text { (Zacharias, 1902) }\end{array}$ & São Paulo/SP & Freshwater (lentic) & - \\
\hline $\begin{array}{l}\text { Chordarium cryptum } \\
\text { Marcus, } 1945\end{array}$ & São Paulo/SP & Freshwater (lentic) & - \\
\hline $\begin{array}{l}\text { Chordarium evelinae } \\
\text { Marcus, } 1945\end{array}$ & São Paulo/SP; Paraná/PR & Freshwater (lentic) & - \\
\hline $\begin{array}{l}\text { Chordarium leucanthum } \\
\text { Marcus, } 1945\end{array}$ & $\begin{array}{c}\text { São Paulo/SP; Paraná/PR; Santo } \\
\text { Antônio da Patrulha/RS }\end{array}$ & Freshwater (lentic) & Mud \\
\hline $\begin{array}{l}\text { Chordarium philum Marcus, } \\
1945\end{array}$ & São Paulo/SP & Freshwater (lentic) & - \\
\hline
\end{tabular}

- No information. 
Table 3. Continued...

\begin{tabular}{|c|c|c|c|}
\hline Species & Locality & Environment & Substratum \\
\hline $\begin{array}{l}\text { Dasyhormus lasius Marcus, } \\
1945\end{array}$ & São Paulo/SP; Paraná/PR & Freshwater (lentic) & - \\
\hline $\begin{array}{l}\text { Dasyhormus lithophorus } \\
\text { Marcus, } 1945\end{array}$ & $\begin{array}{c}\text { São Paulo/SP; Paraná/PR; Santo } \\
\text { Antônio da Patrulha/RS; Camaquã/ } \\
\text { RS }\end{array}$ & Freshwater (lentic) & Mud \\
\hline $\begin{array}{l}\text { Myostenostomum tauricum } \\
\text { Nasonov, } 1924\end{array}$ & $\begin{array}{c}\text { São Paulo/SP; Cachoeirinha/RS; } \\
\text { Santo Antônio da Patrulha/RS; } \\
\text { Camaquã/RS }\end{array}$ & $\begin{array}{l}\text { Humid terrestrial } \\
\text { environment/ } \\
\text { Freshwater (lentic) }\end{array}$ & Mud \\
\hline $\begin{array}{l}\text { Rhynchoscolex evelinae } \\
\text { Marcus, } 1945\end{array}$ & São Paulo/SP & $\begin{array}{l}\text { Freshwater (lentic } \\
\quad \text { and lotic) }\end{array}$ & $\begin{array}{l}\text { On humid } \\
\text { macrophyte leaves } \\
\text { (Dumortiera } \mathrm{sp} .)\end{array}$ \\
\hline $\begin{array}{l}\text { Rhynchoscolex nanus } \\
\text { Marcus, } 1945\end{array}$ & $\begin{array}{c}\text { São Paulo/SP; Curitiba/PR; Santo } \\
\text { Antônio da Patrulha/RS; Camaquã/ } \\
\text { RS }\end{array}$ & Freshwater (lentic) & Mud \\
\hline $\begin{array}{l}\text { Rhynchoscolex platypus } \\
\text { Marcus, } 1945\end{array}$ & $\begin{array}{c}\text { São Paulo/SP; Santo Antônio da } \\
\text { Patrulha/RS }\end{array}$ & Freshwater (lentic) & Mud \\
\hline $\begin{array}{l}\text { Rhynchoscolex pusillus } \\
\text { Marcus, } 1945\end{array}$ & São Paulo/SP & Freshwater (lentic) & Mud \\
\hline $\begin{array}{l}\text { Rhynchoscolex simplex } \\
\text { Leidy, } 1851\end{array}$ & $\begin{array}{c}\text { São Paulo/SP; Terra de Areia/ } \\
\text { RS; Osório/RS; Capivari do Sul/ } \\
\text { RS; Tramandaí/RS; Cachoeirinha/ } \\
\text { RS; Santo Antônio da Patrulha/RS; } \\
\text { Camaquã/RS }\end{array}$ & $\begin{array}{l}\text { Humid terrestrial } \\
\text { environment/ } \\
\text { Freshwater (lentic) }\end{array}$ & Sand/ mud \\
\hline $\begin{array}{l}\text { Stenostomum anatirostrum } \\
\text { Marcus, } 1945\end{array}$ & $\begin{array}{l}\text { São Paulo/SP; Santo Antônio da } \\
\text { Patrulha/RS; Camaquã/RS }\end{array}$ & $\begin{array}{l}\text { Freshwater (lentic } \\
\text { and lotic) }\end{array}$ & Mud \\
\hline $\begin{array}{l}\text { Stenostomum arevaloi } \\
\text { Gieysztor, } 1931\end{array}$ & $\begin{array}{c}\text { São Paulo/SP; Cachoeirinha/RS; } \\
\text { Santo Antônio da Patrulha/RS; } \\
\text { Camaquã/RS }\end{array}$ & $\begin{array}{l}\text { Humid terrestrial } \\
\text { environment/ } \\
\text { Freshwater (lentic) }\end{array}$ & Mud \\
\hline $\begin{array}{l}\text { Stenostomum amphotum } \\
\text { Marcus, } 1945\end{array}$ & $\begin{array}{c}\text { São Paulo/SP; Capivari do Sul/RS; } \\
\text { Cachoeirinha/RS; Santo Antônio da } \\
\text { Patrulha/RS; Camaquã/RS }\end{array}$ & $\begin{array}{l}\text { Humid terrestrial } \\
\text { environment/ } \\
\text { Freshwater (lentic) }\end{array}$ & Mud \\
\hline $\begin{array}{l}\text { Stenostomum bicaudatum } \\
\text { Kennel, } 1888\end{array}$ & $\begin{array}{c}\text { São Paulo/SP; São Leopoldo/ } \\
\text { RS; Novo Hamburgo/RS; Nova } \\
\text { Tramandaí/RS; Santo Antônio da } \\
\text { Patrulha/RS; Terra de Areia/RS; } \\
\text { Osório/RS; Capivari do Sul/RS; } \\
\text { Cachoeirinha/RS; Santo Antônio da } \\
\text { Patrulha/RS; Camaquã/RS }\end{array}$ & $\begin{array}{l}\text { Humid terrestrial } \\
\text { environment/ } \\
\text { Freshwater (lentic) }\end{array}$ & $\begin{array}{l}\text { Mud and sand/ on } \\
\text { macrophyte roots }\end{array}$ \\
\hline $\begin{array}{l}\text { Stenostomum ciliatum } \\
\text { Kepner and Carter, } 1931\end{array}$ & $\begin{array}{c}\text { São Paulo/SP; Paraná/PR; } \\
\text { Cachoeirinha/RS; Santo Antônio da } \\
\text { Patrulha/RS; Camaquã/RS }\end{array}$ & $\begin{array}{l}\text { Humid terrestrial } \\
\text { environment/ } \\
\text { Freshwater (lentic) }\end{array}$ & Mud \\
\hline $\begin{array}{l}\text { Stenostomum corderoi } \\
\text { Marcus, } 1945\end{array}$ & São Paulo/SP & Freshwater (lentic) & - \\
\hline $\begin{array}{l}\text { Stenostomum cryptops } \\
\text { Nuttycombe and Waters, } \\
1935\end{array}$ & São Paulo/SP & Freshwater (lentic) & Bromeliaceae \\
\hline $\begin{array}{l}\text { Stenostomum glandulosum } \\
\text { Kepner and Carter, } 1931\end{array}$ & $\begin{array}{c}\text { São Paulo/SP; } \\
\text { Curitiba/PR; Cachoeirinha/RS. }\end{array}$ & Freshwater (lentic) & Mud \\
\hline
\end{tabular}

- No information. 
Table 3. Continued...

\begin{tabular}{|c|c|c|c|}
\hline Species & Locality & Environment & Substratum \\
\hline $\begin{array}{l}\text { Stenostomum grande Child, } \\
1902\end{array}$ & $\begin{array}{c}\text { São Paulo/SP; Curitiba/PR; São } \\
\text { Leopoldo/RS; Novo Hamburgo/ } \\
\text { RS; Terra de Areia/RS; Capivari do } \\
\text { Sul/RS; Cachoeirinha/RS; Santo } \\
\text { Antônio da Patrulha/RS; Camaquã/ } \\
\text { RS }\end{array}$ & Freshwater (lentic) & $\begin{array}{l}\text { Mud and sand/ on } \\
\text { macrophyte roots }\end{array}$ \\
\hline $\begin{array}{l}\text { Stenostomum } \\
\text { hemisphericum Nasonov, } \\
1924\end{array}$ & Belém/PA & Aquarium & - \\
\hline $\begin{array}{l}\text { Stenostomum leucops } \\
\text { (Duges, 1828) }\end{array}$ & $\begin{array}{c}\text { São Paulo/SP; São Leopoldo/ } \\
\text { RS; Novo Hamburgo/RS; Nova } \\
\text { Tramandaí/RS; Terra de Areia/ } \\
\text { RS; Osório/RS; Tramandaí/RS; } \\
\text { Balneário Pinhal/RS; Capivari do } \\
\text { Sul/RS; Cachoeirinha/RS; Santo } \\
\text { Antônio da Patrulha/RS; Camaquã/ } \\
\text { RS }\end{array}$ & $\begin{array}{l}\text { Humid terrestrial } \\
\text { environment/ } \\
\text { Freshwater (lentic) }\end{array}$ & $\begin{array}{l}\text { Mud and sand/ on } \\
\text { macrophyte roots }\end{array}$ \\
\hline $\begin{array}{l}\text { Stenostomum materazzoi } \\
\text { Marcus, } 1949\end{array}$ & São Paulo/SP & Freshwater (lentic) & Dry pools \\
\hline $\begin{array}{l}\text { Stenostomum membranosum } \\
\text { Kepner and Carter, } 1931\end{array}$ & São Paulo/SP; Cachoeirinha/RS & Freshwater (lentic) & Mud \\
\hline $\begin{array}{l}\text { Stenostomum paraguayense } \\
\text { (Martin, 1908) }\end{array}$ & $\begin{array}{c}\text { São Paulo/SP; Cachoeirinha/RS; } \\
\text { Cachoeirinha/RS; Santo Antônio da } \\
\text { Patrulha/RS; Camaquã/RS }\end{array}$ & $\begin{array}{l}\text { Humid terrestrial } \\
\text { environment/ } \\
\text { Freshwater (lentic) }\end{array}$ & Mud \\
\hline $\begin{array}{l}\text { Stenostomum pegephilum } \\
\text { (Nuttycombe and Waters, } \\
\text { 1938) }\end{array}$ & São Paulo/SP & Freshwater (lotic) & - \\
\hline $\begin{array}{l}\text { Stenostomum } \\
\text { pseudoacetabulum } \\
\text { Nuttycombe and Waters, } \\
1938\end{array}$ & $\begin{array}{c}\text { São Paulo/SP; Cachoeirinha/RS; } \\
\text { Santo Antônio da Patrulha/RS; } \\
\text { Camaquã/RS }\end{array}$ & Freshwater (lentic) & Mud \\
\hline $\begin{array}{l}\text { Stenostomum rosulatum } \\
\text { Marcus, } 1945\end{array}$ & São Paulo/SP & Freshwater (lentic) & Mud \\
\hline $\begin{array}{l}\text { Stenostomum saliens } \\
\text { Kepner and Carter, } 1931\end{array}$ & $\begin{array}{c}\text { São Paulo/SP; } \\
\text { Curitiba/PR; Caiobá/PR } \\
\text { Cachoeirinha/RS; Camaquã/RS }\end{array}$ & Freshwater (lentic) & Mud \\
\hline $\begin{array}{l}\text { Stenostomum simplex } \\
\text { Kepner and Carter, } 1931\end{array}$ & São Paulo/SP & Freshwater (lentic) & Mud \\
\hline $\begin{array}{l}\text { Stenostomum tuberculosum } \\
\text { Nuttycombe and Waters, } \\
1938\end{array}$ & $\begin{array}{c}\text { São Paulo/SP; } \\
\text { Campinas/SP; Cachoeirinha/RS; } \\
\text { Santo Antônio da Patrulha/RS; } \\
\text { Camaquã/RS }\end{array}$ & $\begin{array}{l}\text { Freshwater (lentic } \\
\text { and lotic) }\end{array}$ & Mud \\
\hline $\begin{array}{l}\text { Stenostomum uronephrium } \\
\text { Nuttycombe, } 1931\end{array}$ & São Paulo/SP; Cachoeirinha/RS & Freshwater (lentic) & Mud \\
\hline $\begin{array}{l}\text { Stenostomum } \\
\text { ventronephrium } \\
\text { Nuttycombe, } 1932\end{array}$ & São Paulo/SP & Freshwater (lentic) & - \\
\hline $\begin{array}{l}\text { Stenostomum virginianum } \\
\text { Nuttycombe, } 1931\end{array}$ & $\begin{array}{c}\text { São Paulo/SP; Santa Rita do Passa } \\
\text { Quatro/SP; Paraná/PR }\end{array}$ & $\begin{array}{l}\text { Humid terrestrial } \\
\text { environment/ } \\
\text { Freshwater (lentic) }\end{array}$ & $\begin{array}{l}\text { On humid leaves of } \\
\text { Bromeliaceae/sand }\end{array}$ \\
\hline
\end{tabular}

- No information. 
Table 4. Species of Macrostomida recorded for Brazil, with localities of occurrence and type of environment and/or substratum where the microturbellarians were found.

\begin{tabular}{|c|c|c|c|}
\hline Species & Locality & Environment & Substratum \\
\hline $\begin{array}{l}\text { Archimacrostomum } \\
\text { brasiliensis (Marcus, 1952) }\end{array}$ & $\begin{array}{c}\text { Caraguatatuba/SP; São Sebastião/ } \\
\text { SP }\end{array}$ & Marine & Humid sand \\
\hline $\begin{array}{l}\text { Archimacrostomum } \\
\text { beaufortense (Ferguson, 1937) }\end{array}$ & $\begin{array}{c}\text { Santos/SP; São Vicente/SP; Ilha } \\
\text { das Palmas/SP }\end{array}$ & Marine & Among algae \\
\hline $\begin{array}{l}\text { Austromacrostomum } \\
\text { mortenseni (Marcus, 1950) }\end{array}$ & São Sebastião/SP & Marine & $\begin{array}{c}\text { Coarse sand with Padina } \\
\text { sp. and other algae }\end{array}$ \\
\hline $\begin{array}{l}\text { Karlingia lutheri (Marcus, } \\
\text { 1948) }\end{array}$ & Guarujá/SP; Santos/SP & Marine & $\begin{array}{l}\text { Coarse sand with shell } \\
\text { fragments }\end{array}$ \\
\hline $\begin{array}{l}\text { Macrostomum appendiculatum } \\
\text { Fabricius, } 1826\end{array}$ & $\begin{array}{c}\text { São Sebastião/SP; Caraguatatuba/ } \\
\text { SP }\end{array}$ & Mangrove & Mud \\
\hline $\begin{array}{l}\text { Macrostomum delphax } \\
\text { Marcus, } 1946\end{array}$ & São Paulo/SP; Terra de Areia/RS & Freshwater (lentic) & $\begin{array}{c}\text { On macrophyte } \\
\text { (Eichhornia crassipes) } \\
\text { roots }\end{array}$ \\
\hline $\begin{array}{l}\text { Macrostomum evelinae } \\
\text { Marcus, } 1946\end{array}$ & São Sebastião/SP & Marine & Sand \\
\hline $\begin{array}{l}\text { Macrostomum johni Young, } \\
1972\end{array}$ & $\begin{array}{c}\text { Santo Antônio da Patrulha/ } \\
\text { RS; Osório/RS; Terra de Areia/ } \\
\text { RS; Capivari do Sul/RS; } \\
\text { Cachoeirinha/RS; Santo Antônio } \\
\text { da Patrulha/RS }\end{array}$ & Freshwater (lentic) & $\begin{array}{l}\text { Mud and sand/ on } \\
\text { macrophyte roots }\end{array}$ \\
\hline $\begin{array}{l}\text { Macrostomum tuba Graff, } \\
1882\end{array}$ & $\begin{array}{l}\text { São Paulo/SP; São Leopoldo/ } \\
\text { RS; Nova Petrópolis/RS; Terra de } \\
\text { Areia/RS; Osório/RS; Balneário } \\
\text { Pinhal/RS; Cachoeirinha/RS; } \\
\text { Santo Antônio da Patrulha/RS; } \\
\text { Camaquã/RS }\end{array}$ & Freshwater (lentic) & $\begin{array}{l}\text { Mud and sand/ on } \\
\text { macrophyte roots }\end{array}$ \\
\hline $\begin{array}{l}\text { Macrostomum phocurum } \\
\text { Marcus, } 1954\end{array}$ & São Sebastião/SP; & Mangrove & Mud \\
\hline $\begin{array}{l}\text { Microstomum gabriellae } \\
\text { Marcus, } 1950\end{array}$ & São Sebastião/SP & Marine & $\begin{array}{l}\text { Among algae } \\
\text { (Sargassum sp.) on } \\
\text { rocky coast }\end{array}$ \\
\hline $\begin{array}{l}\text { Microstomum lineare (Müller } \\
\mathrm{OF}, 1773 \text { ) }\end{array}$ & $\begin{array}{c}\text { Terra de Areia/RS; Osório/ } \\
\text { RS; Tramandaí/RS; Capivari } \\
\text { do Sul/RS; Cachoeirinha/RS; } \\
\text { Santo Antônio da Patrulha/RS; } \\
\text { Camaquã/RS }\end{array}$ & Freshwater (lentic) & $\begin{array}{l}\text { Mud and sand/ on } \\
\text { macrophyte roots }\end{array}$ \\
\hline $\begin{array}{l}\text { Microstomum trichotum } \\
\text { Marcus, } 1950\end{array}$ & São Sebastião/SP & Marine & Among calcareous algae \\
\hline $\begin{array}{l}\text { Microstomum ulum Marcus, } \\
1950\end{array}$ & São Sebastião/SP & Marine & $\begin{array}{l}\text { Coarse sand with algae } \\
\text { (Padina sp.) }\end{array}$ \\
\hline $\begin{array}{l}\text { Microstomum breviceps } \\
\text { Marcus, } 1951\end{array}$ & São Sebastião/SP & Marine & $\begin{array}{l}\text { Among algae } \\
\text { (Sargassum } \mathrm{sp} .) \text { on } \\
\text { rocky coast }\end{array}$ \\
\hline $\begin{array}{l}\text { Microstomum rhabdotum } \\
\text { Marcus, } 1951\end{array}$ & São Sebastião/SP & Marine & $\begin{array}{l}\text { Among algae } \\
\text { (Sargassum } \text { sp.) on } \\
\text { rocky coast }\end{array}$ \\
\hline $\begin{array}{l}\text { Myozona evelinae Marcus, } \\
1949\end{array}$ & São Sebastião/SP & Marine & Fine and coarse snad \\
\hline
\end{tabular}


Table 5. Species of Rhabdocoela recorded for Brazil, with localities of occurrence and type of environment and/or substratum where the microturbellarians were found.

\begin{tabular}{|c|c|c|c|}
\hline Species & Locality & Environment & Substratum* \\
\hline \multicolumn{4}{|l|}{ Dalytyphloplanida } \\
\hline Anoplodium evelinae Marcus, 1949 & Santos/SP; São Sebastião/SP & Marine & Holoturoidea \\
\hline Artinga evelinae Marcus, 1948 & Santos/SP; Guarujá/SP & Marine & Among algae \\
\hline Baicalellia evelinae Marcus, 1946 & $\begin{array}{l}\text { São Paulo/SP; Umuarama/SP; } \\
\text { Campos do Jordão/SP }\end{array}$ & Freshwater (lentic) & Sand \\
\hline $\begin{array}{l}\text { Brinkmanniella augusti Marcus, } \\
1951\end{array}$ & São Sebastião/SP & Marine & Among algae \\
\hline $\begin{array}{l}\text { Bothromesostoma evelinae Marcus, } \\
1946\end{array}$ & $\begin{array}{c}\text { São Paulo/SP; Osório/RS; Terra } \\
\text { de Areia/RS. }\end{array}$ & Freshwater (lentic) & Mud \\
\hline $\begin{array}{l}\text { Bothromesostoma personatum } \\
\text { (Schmidt, 1848) }\end{array}$ & $\begin{array}{l}\text { Cachoeirinha/RS; Santo Antônio } \\
\text { da Patrulha/RS; Camaquã/RS }\end{array}$ & Freshwater (lentic) & Mud \\
\hline Byrsophlebs lutheri (Marcus, 1952) & $\begin{array}{c}\text { Cananéia/SP; São Sebastião/SP; } \\
\text { São Vicente/SP }\end{array}$ & Marine & Among algae \\
\hline Daelja secuta Marcus, 1951 & Cananéia/SP & Marine & Areia lodosa \\
\hline Gieysztoria acariaia (Marcus, 1946) & São Paulo/SP & Freshwater (lentic) & - \\
\hline Gieysztoria bellis (Marcus, 1946) & São Paulo/SP & Freshwater (lotic) & $\begin{array}{l}\text { On macrophyte } \\
\text { roots } \\
\text { (Eichhornia } \mathrm{sp} .)\end{array}$ \\
\hline $\begin{array}{l}\text { Gieysztoria complicata (Fuhrmann, } \\
\text { 1912) }\end{array}$ & Curitiba/PR & Freshwater (lentic) & Mud \\
\hline Gieysztoria cypris (Marcus, 1946) & São Paulo/SP & Freshwater (lotic) & $\begin{array}{l}\text { On macrophytes } \\
\text { (Myriophyllum sp.) } \\
\text { and grass }\end{array}$ \\
\hline Gieysztoria evelinae (Marcus, 1946) & São Paulo/SP & Freshwater (lentic) & - \\
\hline Gieysztoria hymanae (Marcus, 1946) & São Paulo/SP & Freshwater (lentic) & - \\
\hline Gieysztoria intricata (Marcus, 1946) & São Paulo/SP & Freshwater (lentic) & Mud \\
\hline Gieysztoria ornata (Hofsten N, 1907) & $\begin{array}{c}\text { São Paulo/SP; Tramandaí/RS; } \\
\text { Osório/RS; Capivari do Sul/RS; } \\
\text { Cachoeirinha/RS }\end{array}$ & Freshwater (lentic) & Mud and sand \\
\hline Gieysztoria rubra (Fuhrmann, 1894) & Cachoeirinha/RS & Freshwater (lentic) & Mud \\
\hline $\begin{array}{l}\text { Gieysztoria therapaina (Marcus, } \\
\text { 1946) }\end{array}$ & Campos do Jordão/SP & Freshwater (lentic) & $\begin{array}{l}\text { On riparian } \\
\text { vegetation }\end{array}$ \\
\hline Gieysztoria thymara (Marcus, 1946) & São Paulo/SP & Freshwater (lotic) & $\begin{array}{l}\text { On macrophytes } \\
\text { (Myriophyllum sp.) } \\
\text { and grass }\end{array}$ \\
\hline Gieysztoria tridesma (Marcus, 1946) & São Paulo/SP; Tremembé/SP & $\begin{array}{l}\text { Freshwater (lentic } \\
\text { and lotic) }\end{array}$ & - \\
\hline $\begin{array}{l}\text { Gieysztoria triquetra (Fuhrmann, } \\
\text { 1894) }\end{array}$ & Terra de Areia/RS & Freshwater (lentic) & $\begin{array}{l}\text { Mud/ on } \\
\text { macrophytes }\end{array}$ \\
\hline Gieysztoria trisolena (Marcus, 1946) & São Paulo/SP; Cachoeirinha/RS & Freshwater (lentic) & Mud \\
\hline Gieysztoria uncia (Marcus, 1946) & São Paulo/SP & Freshwater (lentic) & $\cdot$ \\
\hline $\begin{array}{l}\text { Gyratrix hermaphroditus Ehrenberg, } \\
1831\end{array}$ & $\begin{array}{c}\text { São Paulo/SP; Curitiba/PR; } \\
\text { Terra de Areia/RS; Balneário } \\
\text { Pinhal/RS; Capivari do Sul/RS; } \\
\text { Cachoeirinha/RS; Santo Antônio } \\
\text { da Patrulha/RS; Camaquã/RS }\end{array}$ & Freshwater (lentic) & $\begin{array}{l}\text { Mud and sand/ on } \\
\text { macrophyte roots }\end{array}$ \\
\hline Haloplanella ibla Marcus, 1952 & São Sebastião/SP & Marine & Among algae \\
\hline Kalyla gabriellae Marcus, 1951 & São Vicente/SP; Guarujá/SP & Marine & Sand \\
\hline Lenopharynx triops Marcus, 1951 & São Sebastião/SP & Marine & Among algae \\
\hline Lurus evelinae Marcus, 1950 & Santos/SP & Marine & $\begin{array}{c}\text { Fine sand/ in } \\
\text { intertidal areas }\end{array}$ \\
\hline
\end{tabular}

- No information. * For temnocephalids, the host organism is indicated. 
Table 5. Continued...

\begin{tabular}{|c|c|c|c|}
\hline Species & Locality & Environment & Substratum* \\
\hline $\begin{array}{l}\text { Microdalyellia sawayai Marcus, } \\
1946\end{array}$ & Botucatu/SP; São Paulo/SP & $\begin{array}{l}\text { Freshwater (lotic } \\
\text { and lentic) }\end{array}$ & On macrophytes \\
\hline Memyla phocanella Marcus, 1952 & Caraguatatuba/SP & $\begin{array}{l}\text { Brackish water } \\
\text { (lotic) }\end{array}$ & Muddy sand \\
\hline Mesostoma craci Schmidt, 1858 & Belo Horizonte/MG & Freshwater (lentic) & \\
\hline $\begin{array}{l}\text { Mesostoma ehrenbergii (Focke, } \\
\text { 1836) }\end{array}$ & $\begin{array}{c}\text { São Paulo/SP; Novo Hamburgo/ } \\
\text { RS; Nova Tramandaí/RS; } \\
\text { Terra de Areia/RS; Osório/ } \\
\text { RS; Balneário Pinhal/RS; } \\
\text { Cachoeirinha/RS; Santo Antônio } \\
\text { da Patrulha/RS; Camaquã/RS; } \\
\text { Belo Horizonte/MG }\end{array}$ & Freshwater (lentic) & $\begin{array}{l}\text { Mud and sand/ on } \\
\text { macrophyte roots } \\
\text { and grass }\end{array}$ \\
\hline $\begin{array}{l}\text { Mesostoma lingua (Abildgaard, } \\
1789 \text { ) }\end{array}$ & $\begin{array}{c}\text { Osório/RS; Cachoeirinha/RS; } \\
\text { Santo Antônio da Patrulha/RS; } \\
\text { Camaquã/RS }\end{array}$ & Freshwater (lentic) & Mud \\
\hline $\begin{array}{l}\text { Mesostoma platycephalum Braun, } \\
1885\end{array}$ & $\begin{array}{l}\text { Cachoeirinha/RS; Santo Antônio } \\
\text { da Patrulha/RS; Camaquã/RS }\end{array}$ & Freshwater (lentic) & Mud \\
\hline $\begin{array}{l}\text { Mesostoma productum (Schmidt, } \\
1848 \text { ) }\end{array}$ & $\begin{array}{c}\text { São Leopoldo/RS; Nova } \\
\text { Tramandaí/RS; Nova Petrópolis/ } \\
\text { RS; Terra de Areia/RS; } \\
\text { Osório/RS; Capivari do Sul/RS; } \\
\text { Camaquã/RS }\end{array}$ & Freshwater (lentic) & $\begin{array}{l}\text { Mud and sand/ on } \\
\text { macrophyte roots }\end{array}$ \\
\hline Nygulgus evelinae Marcus, 1954 & Itanhaém/SP & Freshwater (lentic) & Mud \\
\hline $\begin{array}{l}\text { Olisthanella opistomiformis } \\
\text { Nasonov, } 1924\end{array}$ & São Paulo/SP & Freshwater (lentic) & On macrophytes \\
\hline Phaenocora bresslaui Marcus, 1946 & São Paulo/SP & $\begin{array}{l}\text { Freshwater (lentic } \\
\text { and lotic) }\end{array}$ & $\begin{array}{l}\text { On macrophyte } \\
\text { roots (Eichhornia } \\
\text { crassipes) }\end{array}$ \\
\hline $\begin{array}{l}\text { Phaenocora chloroxantha Marcus, } \\
1946\end{array}$ & São Paulo/SP & $\begin{array}{l}\text { Freshwater (lentic } \\
\text { and lotic) }\end{array}$ & $\begin{array}{l}\text { On macrophyte } \\
\text { roots (Eichhornia } \\
\text { crassipes) }\end{array}$ \\
\hline Phaenocora evelinae Marcus, 1946 & São Paulo/SP & $\begin{array}{l}\text { Freshwater (lentic } \\
\text { and lotic) }\end{array}$ & $\begin{array}{l}\text { On macrophyte } \\
\text { roots (Eichhornia } \\
\text { crassipes) }\end{array}$ \\
\hline $\begin{array}{l}\text { Phaenocora typhlops Vejdovsky, } \\
1880\end{array}$ & Cachoeirinha/RS & Freshwater (lentic) & Mud \\
\hline $\begin{array}{l}\text { Phaenocora unipunctata (Ørsted, } \\
1843 \text { ) }\end{array}$ & Cachoeirinha/RS & Freshwater (lentic) & Mud \\
\hline Pogaina suslica (Marcus, 1951) & São Sebastião/SP & Marine & Fine sand \\
\hline Pogaina tifa Marcus, 1954 & São Sebastião/SP & Marine & $\begin{array}{l}\text { Fine and coarse } \\
\text { sand }\end{array}$ \\
\hline Promesostoma scylax Marcus, 1952 & São Sebastião/SP & Marine & Among algae \\
\hline Ruanis pandula Marcus, 1952 & São Sebastião/SP & Marine & Among algae \\
\hline $\begin{array}{l}\text { Strongylostoma dicorymbum Marcus, } \\
1946\end{array}$ & São Paulo/SP & $\begin{array}{l}\text { Freshwater (lentic } \\
\text { and lotic) }\end{array}$ & - \\
\hline $\begin{array}{l}\text { Temnocephala axenos Monticelli, } \\
1889\end{array}$ & $\begin{array}{c}\text { Blumenau/SC; Curitiba/PR; } \\
\text { Porto Alegre/RS; Quaraí/RS; } \\
\text { Rio Grande/RS }\end{array}$ & Freshwater & Decapoda \\
\hline $\begin{array}{l}\text { Temnocephala brevicornis } \\
\text { Monticelli, } 1889\end{array}$ & $\begin{array}{c}\text { Viçosa/MG; Juiz de Fora/MG; } \\
\text { Teresópolis/RJ; Resende/RJ, } \\
\text { Angra dos Reis/RJ; Varginha/SP; } \\
\text { Santa Tereza/ES; Viamão/RS }\end{array}$ & Freshwater (lentic) & Pleurodira \\
\hline $\begin{array}{l}\text { Temnocephala caddisflyi Amato, } \\
\text { Amato and Seixas, } 2011\end{array}$ & Jaboticatubas/MG & Freshwater (lotic) & Trichoptera \\
\hline
\end{tabular}

- No information. * For temnocephalids, the host organism is indicated. 
Table 5. Continued...

\begin{tabular}{|c|c|c|c|}
\hline Species & Locality & Environment & Substratum* \\
\hline $\begin{array}{l}\text { Temnocephala curvicirri Amato and } \\
\text { Amato, } 2005\end{array}$ & Eldorado do Sul/RS; Viamão/RS & Freshwater (lentic) & Hemiptera \\
\hline $\begin{array}{l}\text { Temnocephala cyanoglandula } \\
\text { Amato et al., } 2003\end{array}$ & Cambará do Sul/RS & Freshwater (lentic) & Decapoda \\
\hline $\begin{array}{l}\text { Temnocephala decarloi Moretto, } \\
1978\end{array}$ & $\begin{array}{c}\text { Serra do Cipó/MG; } \\
\text { Brumadinho/MG }\end{array}$ & Freshwater (lentic) & Hemiptera \\
\hline $\begin{array}{l}\text { Temnocephala haswelli Ponce de } \\
\text { Leon, } 1989\end{array}$ & $\begin{array}{c}\text { Porto Alegre/RS; Guaíba/RS; } \\
\text { Eldorado do Sul/RS; Barra } \\
\text { do Ribeiro/RS; Maquiné/RS; } \\
\text { Tramandaí/RS }\end{array}$ & $\begin{array}{l}\text { Freshwater (lentic } \\
\text { and lotic) }\end{array}$ & Mollusca \\
\hline $\begin{array}{l}\text { Temnocephala iheringi Haswell, } \\
1893\end{array}$ & $\begin{array}{c}\text { Porto Alegre/RS; Barra do } \\
\text { Ribeiro/RS; Santa Vitória } \\
\text { do Palmar/RS; Guaíba/RS; } \\
\text { Eldorado do Sul/RS; } \\
\text { Maquiné/RS; } \\
\text { Salobra/MS; Guaicurus/MS }\end{array}$ & $\begin{array}{l}\text { Freshwater (lentic } \\
\text { and lotic) }\end{array}$ & Mollusca \\
\hline $\begin{array}{l}\text { Temnocephala kingsleyae } \\
\text { Damborenea, } 1994\end{array}$ & Pedra do Marcírio/AP & Freshwater (lotic) & Decapoda \\
\hline $\begin{array}{l}\text { Temnocephala lanei Pereira and } \\
\text { Cuoccolo, } 1941\end{array}$ & $\begin{array}{c}\text { Santana do Riacho/MG; } \\
\text { Juquiá/SP. }\end{array}$ & Freshwater (lotic) & Hemiptera \\
\hline $\begin{array}{l}\text { Temnocephala longivaginata } \\
\text { Seixas et al., } 2011\end{array}$ & Peixe Boi/PA & Freshwater (lotic) & Decapoda \\
\hline Temnocephala lutzi Monticelli, 1913 & $\begin{array}{c}\text { Guaíba/RS; Porto Alegre/RS; } \\
\text { Maquiné/RS; São José dos } \\
\text { Ausentes/RS; Rio Negro/AM; } \\
\text { Lago do Prato/AM; Arquipélago } \\
\text { das Anavilhanas/AM; Cachoeira } \\
\text { Grande/AM } \\
\text { Piracicaba/SP; Rio Amapá } \\
\text { Grande/AP }\end{array}$ & $\begin{array}{l}\text { Freshwater (lentic } \\
\text { and lotic) }\end{array}$ & Decapoda \\
\hline $\begin{array}{l}\text { Temnocephala microdactyla } \\
\text { Monticelli, } 1903\end{array}$ & $\mathrm{MG}$ & Freshwater (lotic) & Decapoda \\
\hline $\begin{array}{l}\text { Temnocephala minutocirrus } \\
\text { Amato et al., } 2007\end{array}$ & $\begin{array}{c}\text { Maquiné/RS; São José dos } \\
\text { Ausentes/RS }\end{array}$ & Freshwater (lotic) & Hemiptera \\
\hline $\begin{array}{l}\text { Temnocephala pereirai Volonterio, } \\
2010\end{array}$ & Capão do Leão/RS & Freshwater (lentic) & Pleurodira \\
\hline $\begin{array}{l}\text { Temnocephala pignalberiae Dioni, } \\
1967\end{array}$ & $\begin{array}{c}\text { Poconé/MT; Cuiabá/MT; } \\
\text { Bebedouro/SP }\end{array}$ & $\begin{array}{l}\text { Freshwater (lentic } \\
\text { and lotic) }\end{array}$ & Decapoda \\
\hline $\begin{array}{l}\text { Temnocephala rochensis Ponce de } \\
\text { Leon, } 1979\end{array}$ & $\begin{array}{c}\text { Santa Vitória do Palmar/RS; } \\
\text { Jaguarão/RS; São Lourenço do } \\
\text { Sul/RS }\end{array}$ & $\begin{array}{l}\text { Freshwater (lentic } \\
\text { and lotic) }\end{array}$ & Mollusca \\
\hline $\begin{array}{l}\text { Temnocephala trapeziformis } \\
\text { Amato et al., } 2006\end{array}$ & $\begin{array}{c}\text { Maquiné/RS; São José dos } \\
\text { Ausentes/RS }\end{array}$ & Freshwater (lotic) & Decapoda \\
\hline $\begin{array}{l}\text { Temnocephala travassosfillioi Pereira } \\
\text { and Cuoccolo, } 1941\end{array}$ & $\begin{array}{l}\text { Serra da Cantareira/SP; São } \\
\text { Bernardo do Cipó/SP }\end{array}$ & Freshwater (lotic) & Decapoda \\
\hline Trigonostomum lilliei (Graff, 1911) & Santos/SP; São Vicente/SP & Marine & Among green algae \\
\hline $\begin{array}{l}\text { Trisaccopharynx pusa (Marcus, } \\
\text { 1952) }\end{array}$ & São Sebastião/SP & Marine & Among algae \\
\hline \multicolumn{4}{|l|}{ Kalyptorhynchia } \\
\hline Alcha evelinae Marcus, 1949 & Santos/SP; São Sebastião/SP & Marine & Among algae \\
\hline $\begin{array}{l}\text { Carcharodorhynchus brasiliensis } \\
\text { Reygel, Schockaert and Artois, } 2014\end{array}$ & São Sebastião/SP & Marine & Sand \\
\hline Cheliplana asica Marcus, 1952 & Cananéia/SP; Caraguatatuba/SP & Brackish water & Sand/ mud \\
\hline Cheliplana targa (Marcus, 1952) & Santos/SP & Marine & Fine sand \\
\hline
\end{tabular}

- No information. * For temnocephalids, the host organism is indicated. 
Table 5. Continued...

\begin{tabular}{|c|c|c|c|}
\hline Species & Locality & Environment & Substratum* \\
\hline Harsa obnixa Marcus, 1951 & $\begin{array}{c}\text { Cananéia/SP; Caraguatatuba/SP; } \\
\text { São Sebastião/SP }\end{array}$ & $\begin{array}{l}\text { Freshwater (lotic) } \\
\text { and brackish water }\end{array}$ & Sand/ mud \\
\hline Itaipusa divae Marcus, 1949 & Santos/SP; Praia Grande/SP & Marine & Sand/ on algae \\
\hline Itaipusa evelinae (Marcus, 1954) & São Sebastião/SP; Santos/SP & Marine & $\begin{array}{l}\text { Coarse sand with } \\
\text { algae }\end{array}$ \\
\hline Oneppus timius Marcus, 1952 & $\begin{array}{l}\text { Caraguatatuba/SP; São } \\
\text { Sebastião/SP }\end{array}$ & Marine & Sand/ mud \\
\hline Oneppus lacus Marcus, 1954 & Itanhaém/SP & Marine & Mud \\
\hline $\begin{array}{l}\text { Opisthocystis goettei (Bresslau, } \\
\text { 1906) }\end{array}$ & $\begin{array}{l}\text { Cachoeirinha/RS; Santo Antônio } \\
\text { da Patrulha/RS; Camaquã/RS }\end{array}$ & Freshwater (lentic) & Mud \\
\hline Paulodora felis (Marcus, 1954) & São Sebastião/SP & Marine & Coarse sand \\
\hline Paulodora fredelyna (Marcus, 1948) & Santos/SP & Marine & Among green algae \\
\hline Paulodora matarazzoi Marcus, 1948 & Santos/SP & Marine & Among green algae \\
\hline $\begin{array}{l}\text { Paraustrorhynchus elixus (Marcus, } \\
\text { 1954) }\end{array}$ & Santos/SP; São Sebastião/SP & Marine & Coarse sand \\
\hline Polycystis gabriellae (Marcus, 1948) & Santos/SP; Guarujá/SP & Marine & Among green algae \\
\hline $\begin{array}{l}\text { Proschizorhynchella atopus (Marcus, } \\
\text { 1950) }\end{array}$ & São Sebastião/SP & Marine & Fine sand \\
\hline Rhinolasius sartus Marcus, 1951 & Cananéia/SP & $\begin{array}{l}\text { Freshwater (lentic)/ } \\
\text { brackish water }\end{array}$ & Sand \\
\hline $\begin{array}{l}\text { Schizorhynchoides martae Marcus, } \\
1950\end{array}$ & Cananéia/SP & Brackish & Sand/ mud \\
\hline Toia ycia Marcus, 1952 & São Sebastião/SP & Marine & Among algae \\
\hline $\begin{array}{l}\text { Trapichorhynchus tapes Marcus, } \\
1949\end{array}$ & Santos/SP; São Sebastião/SP & Marine & Coarse sand \\
\hline Utelga deina Marcus, 1949 & Santos/SP & Marine & Among green algae \\
\hline
\end{tabular}

- No information. * For temnocephalids, the host organism is indicated.

Table 6. Species of Prolecithophora, Lecithoepitheliata and Revertospermata recorded for Brazil, with localities of occurrence and type of environment and/or substratum where the microturbellarians were found.

\begin{tabular}{|c|c|c|c|}
\hline Species & Locality & Environment & Substratum \\
\hline \multicolumn{4}{|l|}{ Lecithoepitheliata } \\
\hline $\begin{array}{l}\text { Geocentrophora applanata } \\
\text { (Kennel, 1888) }\end{array}$ & Campinas/SP; Caiobá/PR & $\begin{array}{l}\text { Humid terrestrial } \\
\text { environment/ } \\
\text { Freshwater (lotic) }\end{array}$ & In Bromeliaceae \\
\hline $\begin{array}{l}\text { Prorhynchus stagnalis Schultze, } \\
1851\end{array}$ & $\begin{array}{c}\text { Curitiba/PR; São Paulo/SP; Terra } \\
\text { de Areia/RS; Osório/RS; Capivari } \\
\text { do Sul/RS; Balneário Pinhal/RS; } \\
\text { Tramandaí/RS; Cachoeirinha/RS; } \\
\text { Santo Antônio da Patrulha/RS; } \\
\text { Camaquã/RS }\end{array}$ & $\begin{array}{l}\text { Freshwater } \\
\text { (lentic) }\end{array}$ & $\begin{array}{l}\text { Mud and sand/on } \\
\text { macrophyte roots }\end{array}$ \\
\hline \multicolumn{4}{|l|}{ Prolecithophora } \\
\hline $\begin{array}{l}\text { Acmostomum canarium Marcus, } \\
1947\end{array}$ & Santos/SP & Marine & Among algae \\
\hline $\begin{array}{l}\text { Cylindrostoma netsicum Marcus, } \\
1950\end{array}$ & São Sebastião/SP & Marine & $\begin{array}{l}\text { Among algae } \\
\text { (Sargassum sp.) }\end{array}$ \\
\hline $\begin{array}{l}\text { Cylindrostoma ibeenum Marcus, } \\
1950\end{array}$ & São Sebastião/SP & Marine & Among algae \\
\hline $\begin{array}{l}\text { Cylindrostoma monotrochum } \\
\text { (Graff, 1882) }\end{array}$ & São Sebastião/SP & Marine & $\begin{array}{c}\text { Coarse sand with } \\
\text { Padina sp. and } \\
\text { other algae }\end{array}$ \\
\hline $\begin{array}{l}\text { Cylindrostoma hyljeum Marcus, } \\
1952\end{array}$ & São Sebastião/SP & Marine & Among algae \\
\hline
\end{tabular}

- No information. 
Table 6. Continued...

\begin{tabular}{|c|c|c|c|}
\hline Species & Locality & Environment & Substratum \\
\hline Einarhelmins musta (Marcus, 1952) & Ubatuba/SP & Marine & $\begin{array}{c}\text { Among algae and } \\
\text { bryozoans }\end{array}$ \\
\hline $\begin{array}{l}\text { Monoophorum tigacum Marcus, } \\
1950\end{array}$ & São Sebastião/SP & Marine & Among algae \\
\hline $\begin{array}{l}\text { Plagiostomum acoluthum Marcus, } \\
1948\end{array}$ & Santos/SP & Marine & Among algae \\
\hline $\begin{array}{l}\text { Plagiostomum autectum Marcus, } \\
1948\end{array}$ & Santos/SP & Marine & Among algae \\
\hline $\begin{array}{l}\text { Plagiostomum clusum Marcus, } \\
1951\end{array}$ & São Sebastião/SP & Marine & Coarse sand/ \\
\hline $\begin{array}{l}\text { Plagiostomum elachisterum } \\
\text { (Marcus, 1948) }\end{array}$ & Guarujá/SP; Santos/SP & Marine & Among algae \\
\hline $\begin{array}{l}\text { Plagiostomum evelinae Marcus, } \\
1946\end{array}$ & São Paulo/SP & $\begin{array}{l}\text { Freshwater (lotic } \\
\text { and lentic) }\end{array}$ & - \\
\hline $\begin{array}{l}\text { Plagiostomum girardi girardi } \\
\text { Westblad, } 1956\end{array}$ & Guarujá/SP; Santos/SP & Marine & Among algae \\
\hline $\begin{array}{l}\text { Plagiostomum kurrum Marcus, } \\
1951\end{array}$ & São Sebastião/SP & Marine & Among algae \\
\hline $\begin{array}{l}\text { Plagiostomum lapinum Marcus, } \\
1952\end{array}$ & Caraguatatuba/SP; São Sebastião/SP & Marine & Among algae \\
\hline $\begin{array}{l}\text { Plagiostomum nonatoi Marcus, } \\
1948\end{array}$ & Santos/SP; São Vicente/SP & Marine & $\begin{array}{l}\text { Among green } \\
\text { algae (Ulva sp.) } \\
\text { on rocky coast }\end{array}$ \\
\hline $\begin{array}{l}\text { Plagiostomum remanei Marcus, } \\
1954\end{array}$ & São Sebastião/SP & Marine & $\begin{array}{l}\text { Coarse sand with } \\
\text { Padina } \text { sp. }\end{array}$ \\
\hline Plagiostomum sagax Marcus, 1951 & São Sebastião/SP & Marine & $\begin{array}{l}\text { Coarse sand with } \\
\text { Padina sp. }\end{array}$ \\
\hline $\begin{array}{l}\text { Plagiostomum thelotrichum } \\
\text { Marcus, } 1951\end{array}$ & Santos/SP & Marine & Among algae \\
\hline Plicastoma astrum Marcus, 1947 & Santos/SP & Marine & Sand \\
\hline Plicastoma carvalhoi Marcus, 1947 & Santos/SP & Marine & Among algae \\
\hline Plicastoma phocae Marcus, 1947 & Santos/SP & Marine & Among algae \\
\hline Puzostoma evelinae Marcus, 1950 & Santos/SP; São Sebastião/SP & Marine & Among algae \\
\hline Rosmarium evelinae Marcus, 1950 & São Sebastião/SP & Marine & Among algae \\
\hline Thallagus divae Marcus, 1951 & São Sebastião/SP & Marine & Coarse sand \\
\hline Tuilica evelina Marcus, 1951 & São Sebastião/SP & Marine & Among algae \\
\hline Vorticeros cyrtum Marcus, 1947 & Santos/SP & Marine & Among algae \\
\hline \multicolumn{4}{|l|}{ Revertospermata } \\
\hline Urastoma cyprinae (Graff, 1882) & São Sebastião/SP & Marine & $\begin{array}{c}\text { Among algae, } \\
\text { parasiting a } \\
\text { bivalve }\end{array}$ \\
\hline
\end{tabular}

- No information.

Table 7. Species of Proseriata recorded for Brazil, with localities of occurrence and type of environment and/or substratum where the microturbellarians were found.

\begin{tabular}{|c|c|c|c|}
\hline Species & Locality & Environment & Substratum \\
\hline $\begin{array}{l}\text { Bothrioplana semperi Braun, } \\
1881\end{array}$ & $\begin{array}{l}\text { São Paulo/SP; Santo Antônio } \\
\text { da Patrulha/RS; Camaquã/RS }\end{array}$ & Freshwater (lentic) & $\begin{array}{l}\text { Water with high organic } \\
\text { matter content/ mud }\end{array}$ \\
\hline $\begin{array}{l}\text { Duplominona mica (Marcus, } \\
\text { 1951) }\end{array}$ & São Sebastião/SP & Marine & Sand \\
\hline $\begin{array}{l}\text { Duplominona tridens } \\
\text { (Marcus, 1954) }\end{array}$ & São Sebastião/SP & Marine & $\begin{array}{l}\text { Coarse sand with } \\
\text { Padina sp. }\end{array}$ \\
\hline $\begin{array}{l}\text { Inaloa scalopura (Marcus, } \\
\text { 1949) }\end{array}$ & $\begin{array}{c}\text { São Sebastião/SP; São } \\
\text { Vicente/SP }\end{array}$ & $\begin{array}{c}\text { Freshwater (lotic) and } \\
\text { marine }\end{array}$ & Sand \\
\hline
\end{tabular}


Table 7. Continued...

\begin{tabular}{|c|c|c|c|}
\hline Species & Locality & Environment & Substratum \\
\hline Itaspis evelinae Marcus, 1952 & Ubatuba/SP & Marine & Coarse or medium sand \\
\hline Kata evelinae Marcus, 1949 & São Sebastião/SP & Marine & Sand \\
\hline Kata leroda Marcus, 1950 & São Sebastião/SP & Marine & Fine sand \\
\hline $\begin{array}{l}\text { Meidiama lutheri Marcus, } \\
1946\end{array}$ & Guarujá/SP & & $\begin{array}{l}\text { Water with shell } \\
\text { fragments }\end{array}$ \\
\hline $\begin{array}{l}\text { Mesoda gabriellae Marcus, } \\
1949\end{array}$ & São Sebastião/SP & $\begin{array}{c}\text { Freshwater (lotic) and } \\
\text { marine }\end{array}$ & Sand \\
\hline $\begin{array}{l}\text { Mesoda thelura (Marcus, } \\
\text { 1951) }\end{array}$ & São Sebastião/SP & Marine & Fine sand \\
\hline Minona divae Marcus, 1951 & Rio de Janeiro/RJ & Marine & $\begin{array}{l}\text { Fine sand with shell } \\
\text { fragments }\end{array}$ \\
\hline $\begin{array}{l}\text { Minona evelinae Marcus, } \\
1946\end{array}$ & Guarujá/SP & Marine & $\begin{array}{l}\text { Sand with shell } \\
\text { fragments }\end{array}$ \\
\hline $\begin{array}{l}\text { Monocelis tabira Marcus, } \\
1950\end{array}$ & $\begin{array}{c}\text { Santos/SP; São Sebastião/SP; } \\
\text { Rio de Janeiro/RJ }\end{array}$ & Marine & Fine sand \\
\hline Necia sopha Marcus, 1950 & Santos/SP; São Sebastião/SP & Marine & Among algae \\
\hline $\begin{array}{l}\text { Nematoplana asita Marcus, } \\
1950\end{array}$ & São Sebastião/SP & Marine & Sand \\
\hline $\begin{array}{l}\text { Nematoplana naia Marcus, } \\
1949\end{array}$ & Santos/SP & Marine & Coarse sand \\
\hline $\begin{array}{l}\text { Parotoplana moya Marcus, } \\
1949\end{array}$ & São Sebastião/SP & Marine & Coarse sand \\
\hline $\begin{array}{l}\text { Peraclistus itaipus Marcus, } \\
1950\end{array}$ & Santos/SP & Marine & In tubes of Terebellidae \\
\hline $\begin{array}{l}\text { Philosyrtis eumeca Marcus, } \\
1950\end{array}$ & Santos/SP & Marine & $\begin{array}{l}\text { Fine sand with shell } \\
\text { fragments }\end{array}$ \\
\hline $\begin{array}{l}\text { Promonotus erinaceus } \\
\text { Marcus, } 1950\end{array}$ & São Sebastião/SP & Marine & Sand on a river mouth \\
\hline $\begin{array}{l}\text { Promonotus villacae Marcus, } \\
1949\end{array}$ & São Vicente/SP & $\begin{array}{l}\text { Freshwater (lotic) and } \\
\text { brackish water }\end{array}$ & Sand/ mud \\
\hline $\begin{array}{l}\text { Tabaota curiosa Marcus, } \\
1950\end{array}$ & São Sebastião/SP & Marine & Medium sand \\
\hline $\begin{array}{l}\text { Togarma evelinae Marcus, } \\
1949\end{array}$ & São Sebastião/SP & Marine & Coarse sand \\
\hline $\begin{array}{l}\text { Vannuccia martae Marcus, } \\
1948\end{array}$ & Guarujá/SP & Marine & $\begin{array}{l}\text { Sand with shell } \\
\text { fragments }\end{array}$ \\
\hline $\begin{array}{l}\text { Vannuccia talea Marcus, } \\
1954\end{array}$ & São Sebastião/SP & Marine & $\begin{array}{l}\text { Coarse sand in the } \\
\text { intertidal zone }\end{array}$ \\
\hline
\end{tabular}

\section{Discussion and Conclusions}

Just three Brazilian states, located in southeastern and southern Brazil, concentrate $94 \%$ of the microturbellarian species recorded for the country. Two of them, São Paulo and Rio Grande do Sul, house research groups on turbellarians, clearly reflecting the scientific activity over many years or decades.

Most marine microturbellarian diversity is known only in a small portion of the Warm Temperate Southwestern Atlantic, since the marine microturbellarian groups were almost exclusively studied in a small part of the northern littoral of the state of São Paulo (Marcus, 1948, 1949, 1950, 1952; Hooge and Rocha, 2006). Just scattered records exist for the Tropical Southwestern Atlantic and Northern Brazil Shelf.

From twelve hydrographic regions, only the South Atlantic and Southern Atlantic regions have more than just occasional records of microturbellarians. Such records were mainly distributed among São Paulo, Rio Grande do Sul and Paraná (Marcus, 1943, 1944, 1945a, 1951, 1954; Gamo and Leal-Zanchet, 2004; Vara and Leal-Zanchet, 2013; Braccini and Leal-Zanchet, 2013). Considering the freshwater groups, the temnocephalids certainly constitute an exception, with species recorded for various regions in Brazil (Monticelli, 1899; Pereira and Cuocolo, 1940, 1941; Ferreira Yuki et al., 1993; Damborenea, 1994; Ernst and Lovich, 1996; Vianna and Melo, 2002; Amato et al., 2003, 
2005, 2006, 2007, 2010, 2011; Amato and Amato, 2005; Seixas et al., 2010a, b, c, 2011, 2014). Microturbellarians occurring in terrestrial environments were known only by occasional records in the state of São Paulo (Marcus, 1945b). Considering the six Brazilian biomes, only a small part of the Atlantic Forest, mainly the one located in Southern and Southeastern regions, have records of microturbellarians. Scattered or no records were done for the Amazonian, Caatinga, Savanna, Pampa and Pantanal biomes.

In addition to taxonomical studies, freshwater microturbellarian diversity was also investigated through recent inventories and studies on community structure done in the state of Rio Grande do Sul (Gamo and Leal-Zanchet, 2004; Braccini and Leal-Zanchet, 2013; Vara and Leal-Zanchet, 2013). In these studies, many unidentified species of catenulids, lecithopitheliates, macrostomids and rhabdocoels, among others, were recorded in agroecosystems and natural lentic environments of southern Brazil (Braccini and Leal-Zanchet, 2013; Vara and Leal-Zanchet, 2013). Thus, it is almost unnecessary to emphasize that the known microturbellarian diversity in Brazil represents only a very small part of the existing biodiversity.

Considering the scant information on the taxonomy and biogeography on Brazilian microturbellarians, which is also the situation of the Neotropical microturbellarians in general, some actions should be proposed. First, it would be necessary to sample in the diverse biomes, as well as in the various river and sea basins, following standardized sampling protocols, as for example, the guidelines of the Rapid Assessment Program, in order to quickly generate data about the local and regional diversity (Alonso et al., 2011). Second, it would be necessary to encourage diverse research groups to include microturbellarians and/or turbellarians in general in biodiversity inventories and studies on community structure of invertebrates. Third, it is necessary to increase the number of research groups on microturbellarians, in order to augment the studies on their morphology, systematic, and ecology.

There are, however, some problems for the inclusion of microturbellarians in ample biodiversity inventories. Microturbellarians should be sorted alive under the stereomicroscope and analyzed under the microscope to study their morphology with identification purposes (Young, 2001; Brusa et al., 2003). Thus, some adjustments in the sampling procedures used for other invertebrates are needed, such as the collection of water for sorting the live microturbellarians at the laboratory. Another problem is concerned with the identification of microturbellarians, because for the same reason mentioned above, it would be difficult to send live specimens for the identification at species level by specialists.

Besides the abundance and species richness of microturbellarians in freshwater and marine ecosystems, these organisms may feed on a wide range of benthic and planctonic invertebrates, such as oligochaetes, rotifers, cladocerans, copepods and nematodes, among other organisms including bacteria, algae and protozoans (Young, 2001). Thus, considering their abundance, species richness and ecological importance in aquatic environments, the challenge to study these organisms and enhance their knowledge in Brazilian ecosystems should be faced.

\section{Acknowledgements}

We thank the Brazilian Research Council (CNPq), the Coordenação de Aperfeiçoamento de Pessoal de Nível Superior (CAPES) and Fundação de Amparo à Pesquisa do Estado do Rio Grande do Sul (FAPERGS) for grants and fellowships in support of this study. Juliana Espíndola is acknowlegdeged for her help in summarizing bibliographical information about marine microturbellarians. We thank MSc. Edward Benya and Paulo Firmino for the English review of the manuscript. Dr. Francisco Brusa, Universidad Nacional de La Plata, Argentina, is thanked for a critical reading of an early version of the manuscript. An anonymous reviewer is acknowledged for their helpful suggestions.

\section{References}

ALONSO, L.E., DEICHMANN, J.L., MCKENNA, S.A., NASKRECKI, P. and RICHARDS, S.J., 2011. Biodiversity exploration for conservation: the first 20 years of the Rapid Assessment Program. Arlington: Conservation International. $316 \mathrm{p}$.

AMARAL, S.V., HACK, I.R., ITURRALDE, G.G. and LEALZANCHET, A.M., 2014. Land flatworms (Platyhelminthes: Tricladida) in remnants of deciduous forest in the northeast region of southern Brazil. Biota Neotropica, vol. 14, no. 1, pp. 1-6. http://dx.doi.org/10.1590/S1676-06020140045.

AMATO, J.F.R. and AMATO, S.B., 2005. New species of Temnocephala Blanchard (Platyhelminthes, Temnocephalida) ectosymbiont on giant water bugs, Belostoma spp. (Hemiptera, Belostomatidae) from southern Brazil. Revista Brasileira de Zoologia, vol. 22, no. 1, pp. 107-118. http://dx.doi.org/10.1590/ S0101-81752005000100014.

AMATO, J.F.R., AMATO, S.B. and DAUDT, L.C., 2003. New species of Temnocephala Blanchard (Platyhelminthes, Temnocephalida) ectosymbiont on Aegla serrana Buckup and Rossi (Crustacea, Anomura) from southern Brazil. Revista Brasileira de Zoologia, vol. 20, no. 3, pp. 493-500. http://dx.doi.org/10.1590/ S0101-81752003000300021.

AMATO, J.F.R., AMATO, S.B. and SEIXAS, S.A., 2005. Temnocephala lutzi Monticelli (Platyhelminthes, Temnocephalida) ectosymbiont on two species of Trychodactylus Latreille (Crustacea, Decapoda, Trichodactylidae) from southern Brazil. Revista Brasileira de Zoologia, vol. 22, no. 4, pp. 1085-1094. http:// dx.doi.org/10.1590/S0101-81752005000400038.

AMATO, J.F.R., AMATO, S.B. and SEIXAS, S.A., 2006. A new species of Temnocephala Blanchard (Platyhelminthes, Temnocephalida) ectosymbiont on Trichodactylus fluviatilis Latreille (Crustacea, Decapoda, Trichodactylidae) from southern Brazil. Revista Brasileira de Zoologia, vol. 23, no. 3, pp. 796-806. http://dx.doi.org/10.1590/S0101-81752006000300026.

AMATO, J.F.R., AMATO, S.B., SEIXAS, S.A., FONSECA, M. and ILÁRIO, R.J., 2010. Temnocephala pignalberiae Dioni, 1967 (Platyhelminthes, Temnocephalida) from two allopatric populations of Dilocarcinus pagei Stimpson, 1861 (Crustacea, Decapoda) first record for Brazil. Zootaxa, vol. 2613, pp. 15-28. 
AMATO, J.F.R., AMATO, S.B., SEIXAS, S.A., VIDIGAL, T.H.A. and ANDRADE, C.P., 2011. Trichoptera: the newest insect order host of temnocephalans (Platyhelminthes, Temnocephalida) and the description of a new species of Temnocephala from Brazil. Zootaxa, vol. 2075, pp. 47-58.

AMATO, J.F.R., SEIXAS, S.A. and AMATO, S.B., 2007. A new species of Temnocephala Blanchard (Platyhelminthes, Temnocephalida) ectosymbiont on creeping water bugs, Cryphocricos granulosus De Carlo (Hemiptera, Naucoridae) from southern Brazil. Revista Brasileira de Zoologia, vol. 24, no. 4, pp. 1043-1051. http:// dx.doi.org/10.1590/S0101-81752007000400022.

BAGUÑ̇̀, J. and RIUTORT, M., 2004. The dawn of bilaterian animals: the case of the acoelomorph flatworms. BioEssays, vol. 26, pp. 1046-1057.

BAHIA, J. and PADULA, V., 2009. First record of Pseudoceros bicolor and Pericelis cata (Platyhelminthes: Polycladida) from Brazil. Marine Biodiversity Records, vol. 2, pp. 1-5. http://dx.doi. org/10.1017/S1755267209000918.

BAHIA, J., PADULA, V. and DELGADO, M., 2012. Five new records and morphological data of five polyclad species (Platyhelminthes: Turbellaria) from Rio Grande do Norte, Northeastern Brazil. Zootaxa, vol. 3170, pp. 31-44.

BAMBARADENIYA, C.N.B., EDIRISINGHE, J.P., DE SILVA, D.N., GUNATILLEKE, C.V.S., RANAWANA, K.B. and WIJEKOON, S., 2004. Biodiversity associated with an irrigated rice agroecosystem in Sri Lanka. Biodiversity and Conservation, vol. 13, no. 9, pp. 1715-1753. http://dx.doi. org/10.1023/B:BIOC.0000029331.92656.de.

BAPTISTA, V.A., OLIVEIRA, S.M. and LEAL-ZANCHET, A.M., 2010. Inventário de planárias terrestres (Platyhelminthes, Tricladida) em remanescente de Floresta Estacional Decidual do sul do Brasil. Biota Neotropica, vol. 10, no. 2, pp. 247-252. http:// dx.doi.org/10.1590/S1676-06032010000200027.

BOLL, P.K., ROSSI, I., AMARAL, S.V., OLIVEIRA, S.M., MÜLLER, E.S., LEMOS, V.S. and LEAL-ZANCHET, A.M., 2013. Platyhelminthes ou apenas semelhantes a Platyhelminthes? Relações filogenéticas dos principais grupos de turbelários. Neotropical Biology and Conservation, vol. 8, no. 1, pp. 41-52. http://dx.doi.org/10.4013/nbc.2013.81.06.

BRACCINI, J.A. and LEAL-ZANCHET, A.M., 2013. Turbellarian assemblages in freshwater lagoons in southern Brazil. Invertebrate Biology, vol. 132, no. 4, pp. 305-314. http://dx.doi.org/10.1111/ ivb.12032.

BRUSA, F., DAMBORENEA, C.M. and NOREÑA, C., 2003. A new species of Gieysztoria (Platyhelminthes, Rhabdocoela) from Argentina and a kinship analysis of South American species of the genus. Zoologica Scripta, vol. 32, no. 5, pp. 449-457. http:// dx.doi.org/10.1046/j.1463-6409.2003.00126.x.

BULNES, V.N. and TORRES, Y., 2014. Pseudoceros astrorum, a new species of Polycladida (Cotylea, Pseudocerotidae) from Northeastern Brazil. Zootaxa, vol. 3881, no. 1, pp. 94-100. http:// dx.doi.org/10.11646/zootaxa.3881.1.7. PMid:25543622.

BUSH, L.F., 1981. Marine flora and fauna of the northeastern United States. Turbellaria: Acoela and Nemertodermatida. Seattle: U.S. Dept. of Commerce, National Oceanic and Atmospheric Administration, National Marine Fisheries Service. 71 p. NOAA Technical Report National Marine Fisheries Service Circular, no. 440.

CANNON, L.R.G., 1986. Turbellaria of the World: a guide to families and genera. Brisbane: Queensland Museum. 136 p.
CARBAYO, F. and FROEHLICH, E.M., 2008. Estado do conhecimento dos macroturbelários (Platyhelminthes) do Brasil. Biota Neotropica, vol. 8, no. 4, pp. 177-197. http://dx.doi. org/10.1590/S1676-06032008000400018.

CARBAYO, F., FROEHLICH, E.M., LEAL-ZANCHET, A.M. and AMATO, S.B., 2009. Turbelários (Platyhelminthes). In: R. ROCHA and W. BOEGER, eds. Estado da arte e perspectivas para a zoologia no Brasil. Curitiba: UFPR, pp. 49-64.

DAMBORENEA, M.C., 1994. Temnocefalos neotropicales: Temnocephala kingsleyae sp. n. y T. lutzi Monticelli, 1913 (Platyhelminthes, Temnocephalidea) comensales de crustáceos de Brasil. Iheringia: Série Zoologia, vol. 77, pp. 99-105.

DU BOIS-REYMOND MARCUS, E., 1951. Contributions to the natural history of Brazilian Turbellaria. Comunicaciones Zoologicas del Museo de Historia Natural de Montevideo, vol. 3 , no. 63, pp. 1-25.

DU BOIS-REYMOND MARCUS, E. and MARCUS, E., 1968. Polycladida from Curação and faunistically related regions. Hague: M. Nijhoff. 106 p. Studies on the Fauna of Curaçao and other Caribbean Islands, no. 26.

DUMONT, H.J., RIETZLER, A.C. and HAN, B.P., 2014. A review of typhloplanid flatworm ecology, with emphasis on pelagic species. Inland Waters, vol. 4, no. 3, pp. 257-270. http://dx.doi. org/10.5268/IW-4.3.558.

ERNST, C.H. and LOVICH, J.E., 1996. Hydromedusa maximiliani (Maximilian's Snacke-necked Turtle). Epizoic Comensal. Herpetological Review, vol. 27, no. 2, pp. 76-77.

FERREIRA-YUKI, V.L., DAMBORENEA, M.C. and OSORIOMALLMAN, M.T., 1993. Acantochelys spixii (Duméril et Bibron,1835) (Chelidae) e Trachemys dorbigni (Duméril et Bibron, 1835) (Emydidae) (Testudines) como hospedeiros de Temnocephala brevicornis Monticelli 1889 (Temnocephalidae) (Platyhelminthes). Porto Alegre: PUCRS, pp. 75-83. Comunicações do Museu de Ciências, Série Zoológica, no. 6.

GAMO, J. and LEAL-ZANCHET, A.M., 2004. Freshwater microturbellarians (Platyhelminthes) from Rio Grande do Sul, Brazil. Revista Brasileira de Zoologia, vol. 21, no. 4, pp. 897-903. http://dx.doi.org/10.1590/S0101-81752004000400026.

GAMO, J., 1987. Microturbelarios de la Península Ibérica. Miscelania Zoologica, vol. 11, pp. 41-49.

HOOGE, M.D. and ROCHA, C.E.F., 2006. Acoela (Acoelomorpha) from the northern beaches of the state of São Paulo, Brazil, and a systematic revision of the family Otocelididae. Zootaxa, vol. 1335, pp. 1-50.

HYMAN, L.H., 1951. The invertebrates: Platyhelminthes and Rhynchocoela. New York: McGraw-Hill. The Acoelomate Bilateria.

JENNINGS, J.B., 1997. Nutritional and respiratory pathways to parasitism exemplified in the Turbellaria. International Journal for Parasitology, vol. 27, no. 6, pp. 679-691. http://dx.doi. org/10.1016/S0020-7519(97)00010-6. PMid:9229251.

KOLASA, J., 1991. Flatworms: Turbellaria. In: J.M. THORP and A.P. COVICH, eds. Ecology and classification of North American freshwater invertebrates. New York: Academic Press, vol. 2, pp. 145-171.

LARSSON, K. and JONDELIUS, U., 2008. Phylogeny of Catenulida and support for Platyhelminthes. Organisms, Diversity \& Evolution, vol. 8, no. 5, pp. 378-387. http://dx.doi.org/10.1016/j. ode.2008.09.002. 
LEAL-ZANCHET, A.M., BAPTISTA, V.A., CAMPOS, L.M. and RAFFO, J.F., 2011. Spatial and temporal patterns of land flatworm assemblages in Brazilian Araucaria forests. Invertebrate Biology, vol. 130, no. 1, pp. 25-33. http://dx.doi.org/10.1111/j.17447410.2010.00215.x.

LITTLEWOOD, D.T.J., 2006. The evolution of parasitism in flatworms. In: A.G. MAULE and N.J. MARKS, eds. Parasitic flatworms: molecular biology, biochemistry, immunology and physiology. Wallingford: CAB International, pp. 1-36.

MARCUS, E. and MACNAE, W., 1954. Architomie in a species of Convoluta. Nature, vol. 173, no. 4394, pp. 130. http://dx.doi. org/10.1038/173130a0. PMid:13132888.

MARCUS, E., 1943. O Turbelaria Mesostoma ehrenbergii Focke 1836 no Brasil. Boletim de Indústria Animal, vol. 6, pp. 12-15.

MARCUS, E., 1944. Sobre duas Prorhynchidae (Turbellaria), novas para o Brasil. Arquivos do Museu Paranaense, Curitiba, vol. 4 , no. 1, pp. 3-46.

MARCUS, E., 1945a. Sobre Catenulida Brasileiros. Boletim da Faculdade de Filosofia, Ciências e Letras da Universidade de São Paulo: Série Zoologia, vol. 10, pp. 3-133.

MARCUS, E., 1945b. Sobre microturbellários do Brasil. Montevideo: Museo de Historia Natural. 74 p. Comunicaciones Zoologicas, vol. 1, no. 25.

MARCUS, E., 1946. Sobre Turbellaria brasileiros. Boletim da Faculdade de Filosofia, Ciências e Letras da Universidade de São Paulo: Série Zoologia, vol. 11, p. 1-254.

MARCUS, E., 1948. Turbellaria do Brasil. Boletim da Faculdade de Filosofia, Ciências e Letras da Universidade de São Paulo: Série Zoologia, vol. 13, p. 111-243.

MARCUS, E., 1949. Turbellaria Brasileiros (7). Boletim da Faculdade de Filosofia, Ciências e Letras da Universidade de São Paulo: Série Zoologia, vol. 14, pp. 7-156.

MARCUS, E., 1950. Turbellaria Brasileiros (8). Boletim da Faculdade de Filosofia, Ciências e Letras da Universidade de São Paulo: Série Zoologia, vol. 15, pp. 5-192.

MARCUS, E., 1951. Turbellaria Brasileiros (9). Boletim da Faculdade de Filosofia, Ciências e Letras da Universidade de São Paulo: Série Zoologia, vol. 16, pp. 1-217.

MARCUS, E., 1952. Turbellaria Brasileiros (10). Boletim da Faculdade de Filosofia, Ciências e Letras da Universidade de São Paulo: Série Zoologia, vol. 17, pp. 5-187.

MARCUS, E., 1954. Turbellaria Brasileiros (11). Papéis Avulsos do Departamento de Zoologia e Secretaria de Agricultura de São Paulo, vol. 11, no. 24, pp. 419-489.

MONTICELLI, F.S., 1899. Sulla Temnocephala brevicornis Mont. 1889 e sulle temnocephale en generale. Bollettino della Società dei Naturalisti in Napoli, vol. 12, pp. 72-127.

NOREÑA-JANSSEN, C., 1995. Studies on the taxonomy and ecology of the turbellarian (Plathelminthes) in the floodplain of the Paraná river (Argentina). II. Taxonomy and ecology of the Turbellaria. Archiv für Hydrobiologie, Stuttgart, vol. 107, suppl., pp. 11-262.

PEREIRA, C. and CUOCOLO, R., 1940. Contribução para o conhecimento da morfología, bionomia e ecologia de "Temnocephala brevicornis Monticelli, 1889". Arquivos do Instituto de Biologia do Brasil, vol. 11, pp. 367-398.
PEREIRA, C. and CUOCOLO, R., 1941. Estudos sobre "Temnocephalidae Monticelli, 1899", com estabelecimento de dois novos gêneros australianos e descrição de duas novas espécies neotrópicas. Arquivos do Instituto de Biologia do Brasil, vol. 12, pp. 101-127.

REYGEL, P., SCHOCKAERT, E., JANSSEN, T. and ARTOIS, T., 2014. Two new species of Carcharodorhynchus Meixner, 1938 (Platyhelminthes: Rhabdocoela: Schizorhynchidae) from Brazil and Lanzarote. Marine Biodiversity, vol. 4, pp. 278-285.

RIEGER, R.M., TYLER, S., SMITH, J.P.S. and RIEGER, G.E., 1991. Platyhelminthes: Turbellaria. In: F.W. HARRISON, ed. Microscopic anatomy of invertebrates. New York: Wiley-Liss, vol. 3, pp. 7-140.

ROCHA, O., MATSUMURA-TUNDISI, T., TUNDISI, J.G. and FONSECA, C.P., 1990. Predation on and by pelagic Turbellaria in some lakes in Brazil. Hydrobiologia, vol. 198, no. 1, pp. 91-101. http://dx.doi.org/10.1007/BF00048625.

SCHOCKAERT, E.R., 1996. Turbellarians. In: G.S. HALL, ed. Methods for the examination of organismal diversity in soils and sediments. Wallingford: CAB International, pp. 221-226.

SCHOCKAERT, E.R., HOOGE, M., SLUYS, R., SCHILLING, S., TYLER, S. and ARTOIS, T., 2008. Global diversity of free living flatworms (Platyhelminthes, "Turbellaria") in freshwater. Hydrobiologia, vol. 595, no. 1, pp. 41-48. http://dx.doi.org/10.1007/ s10750-007-9002-8.

SEIXAS, S.A., AMATO, J.F.R. and AMATO, S.B., 2010a. First report of Temnocephala rochensis (Platyhelminthes: Temnocephalida) from Pomace canaliculata (Mollusca: Ampullariidae) outside Uruguay - description updated based on specimens from the State of Rio Grande do Sul, Brazil. Zoologia, vol. 27, no. 5, pp. 820-828. http://dx.doi.org/10.1590/S1984-46702010000500019.

SEIXAS, S.A., AMATO, J.F.R. and AMATO, S.B., 2010b. Redescription of Temnocephala haswelli (Platyhelminthes: Temnocephalida) from Pomacea canaliculata (Mollusca: Ampullariidae) from Brazil: redescription update based on specimens from the state of Rio Grande do Sul, Brazil. Zoologia, vol. 27, no. 3, pp. 455-464. http://dx.doi.org/10.1590/S1984-46702010000300020.

SEIXAS, S.A., AMATO, J.F.R. and AMATO, S.B., 2010c. Redescription of Temnocephala iheringi (Platyhelminthes: Temnocephalida) based on specimens from Pomacea canaliculata (Mollusca: Ampullariidae) of the state of Rio Grande do Sul, Brasil: the possible type host and type locality. Zoologia, vol. 27, no. 2, pp. 245-257. http://dx.doi.org/10.1590/S1984-46702010000200012.

SEIXAS, S.A., AMATO, J.F.R. and AMATO, S.B., 2011. A new species of Temnocephala Blanchard (Platyhelminthes, Temnocephalida) ectosymbiont on Dilocarcinus septemdentatus (Decapoda, Trichodactylidae) from the Brazilian Amazonia. Neotropical Helminthology, vol. 5, pp. 200-211.

SEIXAS, S.A., AMATO, J.F.R., AMATO, S.B. and MASCARENHAS, C.S., 2014. First report of Temnocephala pereirai (PLatyhelminthes, Temnocephalidae) on Trachemys dorbigni (Embyididae) from Southern Brazil: a complete morphological study. Neotropical Helminthology, vol. 8, pp. 23.

SPALDING, M.D., FOX, H.E., ALLEN, G.R., DAVIDSON, N., FERDAÑA, Z.A., FINLAYSON, M., HALPERN, B.S., JORGE, M.A., LOMBANA, A.L., LOURIE, S.A., MARTIN, K.D., MCMANUS, E., MOLNAR, J., RECCHIA, C.A. and ROBERTSON, J., 2007. Marine ecoregions of the world: a bioregionalization of coastal and shelf areas. Bioscience, vol. 57, no. 7, pp. 573-583. http://dx.doi.org/10.1641/B570707. 
TESSENS, B., JANSSEN, T. and ARTOIS, T., 2014. Molecular phylogeny of Kalyptorhynchia (Rhabdocoela, Platyhelminthes) inferred from ribosomal sequence data. Zoologica Scripta, vol. 43, no. 5, pp. 519-530. http://dx.doi.org/10.1111/zsc.12066.

TYLER, S., SCHILLING, S., HOOGE, M. and BUSH, L.F., 2014 [viewed 10 October 2014]. Turbellarian taxonomic database. Version 1.7 [online]. Orono: University of Maine. Available from: http://turbellaria.umaine.edu

VAN STEENKISTE, N., TESSENS, B., WILLEMS, W., BACKELJAU, T., JONDELIUS, U. and ARTOIS, T., 2013. A comprehensive molecular phylogeny of Dalytyphloplanida (Platyhelminthes: Rhabdocoela) reveals multiple escapes from the marine environment and origins of symbiotic relationships.
PLoS One, vol. 8, no. 3, pp. e59917. http://dx.doi.org/10.1371/ journal.pone.0059917. PMid:23536894.

VARA, D.C. and LEAL-ZANCHET, A.M., 2013. Turbelários límnicos (Platyhelminthes) em ecossistemas de arroz irrigado da Planície Costeira do sul do Brasil. Biota Neotropica, vol. 13, no. 4, pp. 1-11. http://dx.doi.org/10.1590/S1676-06032013000400021.

VIANNA, G J.C. and MELO, A.L., 2002. Aquatic Heteroptera as host of Temnocephala Blanchard (Platyhelminthes: Temnocephalidae) in Minas Gerais, Brazil. Lundiana, vol. 3, pp. 151-153.

YOUNG, J.O., 2001. Keys to the freshwater microturbellarians of Britain and Ireland with notes on their ecology. Ambleside: The Freshwater Biological Association. 142 p. Freshwater Biological Association Scientific Publication, no. 59. 